\title{
THE Q/U IMAGING EXPERIMENT: POLARIZATION MEASUREMENTS OF RADIO SOURCES AT
} 43 AND $95 \mathrm{GHz}$

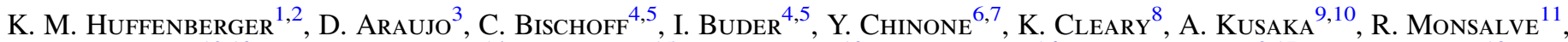 \\ S. K. NÆss ${ }^{12,13}$, L. B. Newburgh ${ }^{14}$, R. Reeves ${ }^{15}$, T. M. Ruud ${ }^{13}$, I. K. Wehus ${ }^{16}$, J. T. L. Zwart ${ }^{3,17}$, C. Dickinson $^{18}$, \\ H. K. Eriksen ${ }^{13,19}$, T. Gaier ${ }^{16}$, J. O. Gundersen ${ }^{2}$, M. Hasegawa ${ }^{6}$, M. Hazumi ${ }^{6}$, A. D. Miller ${ }^{3}$, S. J. E. Radford ${ }^{8}$, \\ A. C. S. Readhead ${ }^{8}$, S. T. Staggs ${ }^{10}$, O. Tajima ${ }^{4,6}$, and K. L. Thompson ${ }^{20}$ \\ (QUIET COLlaboration) \\ ${ }^{1}$ Department of Physics, Florida State University, P.O. Box 3064350, Tallahassee, FL 32306-4350, USA; huffenbe@ physics.fsu.edu \\ ${ }^{2}$ Department of Physics, University of Miami, 1320 Campo Sano Drive, Coral Gables, FL 33146, USA \\ ${ }^{3}$ Department of Physics and Columbia Astrophysics Laboratory, Columbia University, New York, NY 10027, USA \\ ${ }^{4}$ Kavli Institute for Cosmological Physics, Department of Physics, Enrico Fermi Institute, The University of Chicago, Chicago, IL 60637, USA \\ ${ }^{5}$ Harvard-Smithsonian Center for Astrophysics, 60 Garden Street MS 42, Cambridge, MA 02138, USA \\ ${ }^{6}$ High Energy Accelerator Research Organization (KEK), 1-1 Oho, Tsukuba, Ibaraki 305-0801, Japan \\ Department of Physics, University of California, Berkeley, CA 94720, USA \\ ${ }^{8}$ Cahill Center for Astronomy and Astrophysics, California Institute of Technology, 1200 E. California Blvd M/C 249-17, Pasadena, CA 91125, USA \\ ${ }^{9}$ Physics Division, Lawrence Berkeley National Laboratory, 1 Cyclotron Road, Berkeley, CA 94720, USA \\ ${ }^{10}$ Joseph Henry Laboratories of Physics, Jadwin Hall, Princeton University, Princeton, NJ 08544, USA \\ ${ }^{11}$ School of Earth and Space Exploration, Arizona State University, 781 E. Terrace Road, Tempe, AZ 85287, USA \\ ${ }^{12}$ Department of Astrophysics, University of Oxford, Keble Road, Oxford, OX1 3RH, UK \\ ${ }^{13}$ Institute of Theoretical Astrophysics, University of Oslo, P.O. Box 1029 Blindern, NO-0315 Oslo, Norway \\ ${ }^{14}$ Dunlap Institute, University of Toronto, 50 St. George Street, Toronto, ON M5S 3H4, Canada \\ ${ }^{15}$ CePIA, Departamento de Astronomía, Universidad de Concepción, Chile \\ 16 Jet Propulsion Laboratory, California Institute of Technology, 4800 Oak Grove Drive, Pasadena, CA 91109, USA \\ ${ }_{17}^{17}$ Physics Department, University of the Western Cape, Private Bag X17, Bellville 7535, South Africa \\ 18 Jodrell Bank Centre for Astrophysics, Alan Turing Building, School of Physics and Astronomy, \\ The University of Manchester, Oxford Road, Manchester M13 9PL, UK \\ ${ }^{19}$ Centre of Mathematics for Applications, University of Oslo, P.O. Box 1053 Blindern, NO-0316 Oslo, Norway \\ ${ }^{20}$ Kavli Institute for Particle Astrophysics and Cosmology and Department of Physics, Stanford University, \\ Varian Physics Building, 382 Via Pueblo Mall, Stanford, CA 94305, USA \\ Received 2014 November 24; accepted 2015 April 17; published 2015 June 10
}

\begin{abstract}
We present polarization measurements of extragalactic radio sources observed during the cosmic microwave background polarization survey of the Q/U Imaging Experiment (QUIET), operating at $43 \mathrm{GHz}$ (Q-band) and $95 \mathrm{GHz}$ (W-band). We examine sources selected at $20 \mathrm{GHz}$ from the public, $>40 \mathrm{mJy}$ catalog of the Australia Telescope (AT20G) survey. There are $~ 480$ such sources within QUIET's four low-foreground survey patches, including the nearby radio galaxies Centaurus A and Pictor A. The median error on our polarized flux density measurements is $30-40 \mathrm{mJy}$ per Stokes parameter. At signal-to-noise ratio $>3$ significance, we detect linear polarization for seven sources in Q-band and six in W-band; only $1.3 \pm 1.1$ detections per frequency band are expected by chance. For sources without a detection of polarized emission, we find that half of the sources have polarization amplitudes below $90 \mathrm{mJy}$ (Q-band) and $106 \mathrm{mJy}$ (W-band), at 95\% confidence. Finally, we compare our polarization measurements to intensity and polarization measurements of the same sources from the literature. For the four sources with WMAP and Planck intensity measurements $>1 \mathrm{Jy}$, the polarization fractions are above 1\% in both QUIET bands. At high significance, we compute polarization fractions as much as $10 \%-20 \%$ for some sources, but the effects of source variability may cut that level in half for contemporaneous comparisons. Our results indicate that simple models — ones that scale a fixed polarization fraction with frequency - are inadequate to model the behavior of these sources and their contributions to polarization maps.
\end{abstract}

Key words: cosmic background radiation - galaxies: active - galaxies: individual (Cen A, Pict A) - methods: statistical - polarization

\section{INTRODUCTION}

Polarized cosmic microwave background $(\mathrm{CMB})$ fluctuations are important for cosmological analysis because they carry information that is complementary to temperature fluctuations, and they can therefore tighten cosmological parameter constraints. Furthermore, observations of the large-scale odd-parity ( $B$-mode) polarization patterns predicted by inflation can constrain inflationary models and, consequently, the underlying GUT-scale physics. ${ }^{21} \mathrm{On}$

\footnotetext{
${ }^{21}$ Upward revisions in the assessment of the Galactic dust contamination (Planck Collaboration Int. XXX et al. 2014) have tempered initial claims of CMB $B$-mode observations (BICEP2 Collaboration 2014).
}

small scales, polarization fluctuations are essential to establish high signal-to-noise ratio $(\mathrm{S} / \mathrm{N})$ measurements of $\mathrm{CMB}$ lensing caused by foreground structure (Hirata \& Seljak 2003; Okamoto \& Hu 2003; Smith et al. 2012). However, polarized emission-from active galactic nuclei (AGNs) and other extragalactic sourcescreates an additional source of fluctuations in the polarized microwave sky, leading to a small-scale systematic effect for CMB polarization experiments (e.g., de Zotti et al. 1999, 2005; Tucci et al. 2004, 2005, 2011; The COrE Collaboration et al. 2011; Tucci \& Toffolatti 2012). Polarized point sources will limit bispectrum non-Gaussianity parameter $f_{\mathrm{NL}}$ studies at lower $\mathrm{CMB}$ observation frequencies, $\nu<100 \mathrm{GHz}$ (Curto et al. 2013). 
Measurements of bright polarization sources help us to refine models of the point source emission, and account for the contribution of dimmer, unobserved sources. Also, if we can identify bright and compact polarized sources, they make valuable calibrators for millimeter wave polarization surveys (e.g., Agudo et al. 2012).

In the following we make polarization measurements of extragalactic radio sources at 43 and $95 \mathrm{GHz}$ with data from the Q/U Imaging ExperimenT (QUIET Collaboration et al. 2011, 2012, 2013). This work is ancillary to QUIET's main aim to measure the CMB polarization, but useful because these measurements provide information about the cores and jets of active galaxies, and they provide a handle on the contamination by such sources to measurements of the CMB (Mesa et al. 2002; López-Caniego et al. 2009; Jackson et al. 2010; Battye et al. 2011).

In unification models of active galaxies, objects that look different may actually be similar, containing central, supermassive black holes with accretion disks and jets of relativistic plasma (Readhead et al. 1978; Begelman et al. 1984; Antonucci 1993; Urry \& Padovani 1995). Luminosities and spectral lines may differ simply because of the object's angle to the line of sight. In addition, systems may differ in black hole mass, angular momentum, or accretion rate, or possess different interstellar media. These objects radiate across the electromagnetic spectrum via synchrotron and inverse Compton emission, including at millimeter wavelengths $(30-300 \mathrm{GHz})$. At frequencies $\geqslant 1 \mathrm{GHz}$, they typically have polarization fractions of a few percent (Stil et al. 2014). Because they are so luminous, we can use them to watch the evolution of supermassive black holes and their host galaxies over cosmic time.

An advantage of millimeter observations of AGN polarization is that they avoid the undesirable effect of Faraday rotation. The angle of Faraday rotation $(\beta)$ is proportional to the square of the wavelength, $\beta=\mathrm{RM} \lambda^{2}$, and the typical rotation measures $(\mathrm{RM})$ in these objects are $\sim 10^{2}-10^{4} \mathrm{rad} \mathrm{m}^{-2}$ (Zavala \& Taylor 2002, 2003, 2004). Thus only at wavelengths shorter than $\lambda \sim 10^{-3} \mathrm{~m}$ can we reduce Faraday rotation (and the subsequent depolarization in regions of inhomogeneous magnetic field) to levels that allow order-degree measurements of the intrinsic polarization direction, even for bright sources. Also, at longer wavelengths, the jet's opacity shrouds its inner parts due to synchrotron self-absorption, while at millimeter wavelengths, measurements like these probe the magnetic field environment in the inner jet regions (Potter \& Cotter 2012).

However, as of today there are only a few polarization surveys of AGNs $>20 \mathrm{GHz}$, particularly at $<1 \mathrm{Jy}$, due to observational challenges. Compared to radio telescopes, millimeter-wave telescopes and receivers are less sensitive and the sources are often dimmer. Efforts include those by Agudo et al. (2010), who targeted 145 sources with the Institut de Radioastronomie Millimétrique (IRAM) $30 \mathrm{~m}$ dish at $90 \mathrm{GHz}$; López-Caniego et al. (2009), who identified 22 objects in WMAP polarization data (Page et al. 2007), and Battye et al. (2011), who made VLA measurements at 8-43 GHz of WMAP catalog sources (Wright et al. 2009).

Therefore, much of the information about AGNs at these frequencies comes from $\mathrm{CMB}$ surveys themselves. Although dedicated CMB experiments often target angular scales that are significantly larger than $1^{\prime}$, they nonetheless make useful contributions to AGN science because they survey large areas of the sky, rather than aiming at known targets. QUIET's unique dataset, one of the most sensitive to date at these frequencies, will at Q-band continue to be competitive even after the Planck polarization data release. QUIET's beams have FWHMs of $27^{\prime}$ and $13^{\prime}$, large compared to most AGNs, so for all but a few systems we measure an integrated flux density for the whole system.

Most AGNs, particularly blazars, are known to be variable, so it can be useful to make many short-duration observations with multiple bands simultaneously (Agudo et al. 2014). By contrast, the long duration campaigns for some CMB observation strategies may provide only season- or year-long average emission. $^{22}$ This includes our QUIET observations, as the Q- and W-band observations took place in subsequent years, 2008-2009 (8 months) and 2009-2010 (17 months). The corresponding maps integrate observations over whole seasons to achieve low noise over broad sky areas, which is important for $\mathrm{CMB}$ measurements. Other on-going lowfrequency $\mathrm{CMB}$ and $\mathrm{CMB}$-foreground experiments, like C-BASS (5 GHz, 44' FWHM; Holler et al. 2011; King et al. 2014) and QUIJOTE (10-40 GHz, 17'-55' FWHM; RubiñoMartín et al. 2012), can also provide useful AGN scientific results.

Our method probes the polarization of objects with known positions. Typically CMB surveys are less sensitive in polarization than total intensity, and the sources are often just a few percent polarized. It is therefore useful to detect sources in total intensity, and also use those same measurements to determine the source positions. Alternatively, one could take source positions from an external catalog or different frequency bands, where a particular instrument may be more sensitive or the sources are brighter. For AGNs, radio surveys like NVSS and FIRST (and the VLA Sky Survey ${ }^{23}$ in the future) can identify sources down to sub-mJy flux levels, and have small positional uncertainty (Becker et al. 1995; Condon et al. 1998; Murphy \& Baum 2014). The use of external source catalogs is particularly important for QUIET, because the QUIET detector technology is specifically optimized for dedicated polarization measurements, and so deep QUIET temperature maps are not readily available.

In this work, we adopt the catalog from the Australia Telescope $20 \mathrm{GHz}$ (AT20G) Survey of the Southern sky (Massardi et al. 2008; Murphy et al. 2010), and we measure the polarized flux density at the specified locations in the QUIET sky maps, carefully accounting for the errors caused by instrumental noise and background CMB fluctuations. We then compare the measured fluxes in the two QUIET bands to intensity and polarization measurements of the same sources in the literature.

In what follows, we first, in Section 2, describe the QUIET observations and the AT20G source sample. Then in Section 3, we outline the method for measuring the polarized source flux density from our QUIET maps, before reporting our results in Section 4. Finally, in Section 5 we present our conclusions.

Throughout we refer to Stokes parameters defined in Galactic coordinates, using the convention common in mathematical and CMB literature (e.g., Zaldarriaga \&

\footnotetext{
22 For example, WMAP scanned large areas of the sky rapidly and repeatedly, building up sensitivity gradually. Planck, by contrast, focused on one thin ring on the sky at a time, achieving the full survey sensitivity after a few minutes, but not returning to the same region of sky until the next survey, months later. ${ }^{23} \mathrm{https}: / /$ science.nrao.edu/science/surveys/vlass
} 
Seljak 1997). This facilitates direct comparison to Planck and $W M A P C M B$ maps at the same frequencies, which are sensitive to sources down to $\sim 1 \mathrm{Jy}$. We use $Q$ and $U$ to refer to Stokes parameter values in the CMB map in thermodynamic temperature. Flux density is denoted by $S_{Q}$ and $S_{U}$. For the polarization amplitude we similarly use $P$ and $S_{P}$, and for the polarization angle we use $\alpha$.

The Stokes parameters and linear polarized flux density are related by

$$
\begin{aligned}
S_{P} & =\left(S_{Q}^{2}+S_{U}^{2}\right)^{1 / 2} \\
\alpha & =\frac{1}{2} \arctan \left(S_{U} / S_{Q}\right) \\
S_{Q} & =S_{P} \cos (2 \alpha) \\
S_{U} & =S_{P} \sin (2 \alpha)
\end{aligned}
$$

where $\alpha$ is the polarization angle between the electric field and a fiducial axis, and care is taken with the signs in the arctangent to get the angle in the correct quadrant. The pairs of quantities $\left(S_{Q}, S_{U}\right)$ and $\left(S_{P}, 2 \alpha\right)$ are equivalent to Cartesian and polar coordinates in a plane, ${ }^{24}$ which aids us in later computations.

In this convention, the polarization angle is measured east of south, and differs from the IAU convention, where the polarization angle is measured east of north, so $\alpha_{\mathrm{IAU}}=\pi-\alpha$. This causes IAU convention to differ in the sign of $U$, so that $S_{U, \mathrm{IAU}}=-S_{U}$ as we have written it (Górski et al. 2005). Since IAU convention is more common for position angles, we use it in our tables for bright sources (Tables 3 and 4), and we further rotate from Galactic to Celestial coordinates to facilitate comparisons with other AGN measurements. Source positions and angles are in the J2000 epoch.

\section{DATA}

\subsection{QUIET CMB Maps}

The QUIET instrument (QUIET Collaboration et al. 2013) consisted of a $1.4 \mathrm{~m}$ side-fed Dragonian telescope coupled to an array of correlation polarimeters. These used High Electron Mobility Transistor (HEMT) amplifiers cooled to $20 \mathrm{~K}$. Situated on the Chajnantor plateau in Chile's Atacama region, QUIET made polarized microwave measurements of six patches of the sky, two in the Galactic plane, and four in low-foreground regions. The latter yielded polarization maps and CMB power spectra at $43 \mathrm{GHz}$ (Q-band, QUIET Collaboration et al. 2011) and $95 \mathrm{GHz}$ (W-band, QUIET Collaboration et al. 2012).

The Q-band receiver had 17 polarization-sensitive assemblies with a total sensitivity of $69 \mu \mathrm{Ks}^{1 / 2}$, and was mounted on the telescope during the first observing season beginning in 2008 October. The W-band receiver had 84 polarizationsensitive assemblies and a sensitivity of $87 \mu \mathrm{Ks}^{1 / 2}$, and was mounted on the telescope during 2009 July. The second observing season proceeded until 2010 December. The instrument produced more than $10,000 \mathrm{hr}$ of data between the two bands. For point sources in the low-foreground patches (totaling $\sim 1000 \mathrm{deg}^{2}$ ), this yields a median $1 \sigma$ sensitivity per Stokes parameter of $32 \mathrm{mJy}$ for Q-band and $39 \mathrm{mJy}$ for Wband. Due to the detector technology, most QUIET modules

\footnotetext{
${ }^{24}$ In optics, the mid-plane of the Poincaré sphere.
}

were sensitive to polarization only. A small number of temperature-sensitive modules provided data about the atmosphere or the beams (during planet mapping), but QUIET does not produce deep temperature maps over the survey regions.

Bandpasses for QUIET were determined with a signal generator and standard gain horn, injecting known signals into the front window of the cryostat. Effective frequencies in polarization were found to be 43.0 and $94.4 \mathrm{GHz}$ for Q- and Wbands, respectively, with 7.6 and $10.7 \mathrm{GHz}$ bandwidths, for a CMB blackbody spectrum (Tables 6 and 11, QUIET Collaboration et al. 2013). Below we adopted the CMB values for our computation of flux density. Note that for AGN-type spectra $^{25}$ the effective frequencies are lower by $\sim 0.2$ and $0.1 \mathrm{GHz}$, respectively, but at our relatively low $\mathrm{S} / \mathrm{N}$, this difference is not important. For a source with a rotation measure of $10^{3} \mathrm{rad} \mathrm{m}^{-2}$, we would expect Faraday rotation at the center of Q-band of 2:8, with roughly a degree difference between the low- and high-frequency ends of the band. For the center of W-band, the rotation would be 0.6 , with less than two-tenths of a degree difference between the ends of the bands.

For both arrays, we derived beam profiles from observations of Jupiter and Tau A, the brightest unpolarized and polarized sources on the sky that are compact compared to the QUIET beams. Observations with the polarimeter modules of the fainter polarization signal from Tau A were found to be consistent with the Jupiter profiles from the temperature modules, after accounting for bandpasses, source spectra, and horn positions within the focal plane (QUIET Collaboration et al. 2013).

The impact of the beam on the map is nearly axisymmetric, a product of the intrinsic roundness of the optical beams, sky rotations during observing, and scheduled, physical rotations of the telescope about its boresight. Including the effect of scanto-scan pointing jitter, the FWHM of the effective beam for Qband is $27: 3$; for W-band it is $12 ! 8$. We compute the beam window function with a Legendre transform of a onedimensional Hermite expansion of the symmetrized beam (Monsalve 2010; QUIET Collaboration et al. 2013). A pixel window function captures the integration over the finite-sized pixels. This is folded into our expected profile for a point-like source. Any error in pointing reduces the measured source flux density. The uncertainty on the absolute pointing calibration is 3.5 for Q-band; in simulations, such an error in pointing decreases the measured flux from a source by about $2.1 \%$, much smaller than our other errors. For W-band, the uncertainty is $2^{\prime}$, but the beam is smaller, and this position error decreases the measured flux by $11 \%$ in simulations. For our bright sources $(\sim 100 \mathrm{mJy})$, this is less than a third of the typical statistical error, but for most sources it is much less.

We characterized the instrumental polarization with Jupiter, decomposing the polarization leakage maps into GaussHermite moments (Monsalve 2010; QUIET Collaboration et al. 2013). The largest moment was the Q-band temperature to polarization monopole, for which the module-median leakage was $\sim 1 \%$. The $\mathrm{W}$-band median leakage was $0.25 \%$, and typical values for the higher moments ranged between $0.2 \%$ and $0.4 \%$. Sky rotation and instrumental rotation around the boresight help to mitigate this leakage. For instance, in the W-band power spectrum analysis (QUIET Collaboration et al.

\footnotetext{
25 The typical AGN-type spectrum at microwave frequencies is nearly flat, with $S \propto \nu^{\alpha \text { SED }}$ and $\alpha_{\mathrm{SED}} \sim 0$.
} 
Table 1

Summary of QUIET Source Observations

\begin{tabular}{lcccc}
\hline \hline Band & $\begin{array}{c}\nu \\
(\mathrm{GHz})\end{array}$ & $\begin{array}{c}\text { FWHM } \\
(\operatorname{arcmin})\end{array}$ & $\begin{array}{c}\text { Pol. Sensitivity } \\
(\mathrm{mJy})\end{array}$ & $\begin{array}{c}\text { Count } \\
(\mathrm{S} / \mathrm{N}>3)\end{array}$ \\
\hline $\mathrm{Q}$ & 43.0 & 27.3 & $32 \mathrm{mJy}$ & 7 \\
$\mathrm{~W}$ & 94.4 & 12.8 & $39 \mathrm{mJy}$ & 6 \\
\hline
\end{tabular}

Note. For each band, the effective frequency, beam FWHM, median polarization sensitivity to point sources with known position (per Stokes parameter), and the number of compact sources in which we detect polarized emission at $\mathrm{S} / \mathrm{N}>3$ (defined by Equation (6)).

2012), these effects yielded a factor of $\sim 40$ improvement in the systematic $B B$ contamination (assuming zero input $B$-modes signal) and a factor of $\sim 10$ improvement for $E E .^{26}$ For Q-band, with less variety in rotation about the telescope boresight, the mitigation degrades by roughly a factor 1.3 in the power spectrum.

To control systematic errors, the QUIET analysis used a pair of map-making and power spectrum estimation pipelines. This paper uses products from the maximum likelihood pipeline (pipeline " $\mathrm{B}$ " in earlier papers), which projects the timeordered data into the map domain while accounting for its noise covariance, the telescope pointing model, and a bandpass filter that suppressed both low frequency noise and scan-synchronous contamination. In addition to the actual map, the maximum-likelihood method also produces a full pixel-pixel noise covariance, which forms the noise model adopted below. From these maps and covariance matrices, we excise miniature maps containing only those pixels within a radius of $60^{\prime}$ (Qband) or $30^{\prime}$ (W-band) of the source catalog positions, depending on the band's beam size, and we retain only the pixel noise covariance for these small sub-maps. Finally, because of the high-pass filters applied in the original QUIET analysis, the very largest scales in these maps are associated with significant uncertainties, and we therefore marginalize over a baseline offset for each source, adopting independent offset planes in the Stokes $Q$ and $U$ parameters.

Table 1 summarizes our observations for point sources, which we detail below.

\subsection{AT20G Source Sample}

The Australia Telescope $20 \mathrm{GHz}$ Survey (AT20G, Massardi et al. 2008, 2011b; Murphy et al. 2010) covered sources across the entire southern hemisphere. The source catalog includes 5890 sources brighter than $40 \mathrm{mJy}$ at $20 \mathrm{GHz}$. For all sources in the catalog, they report $\mathrm{S} / \mathrm{N} \geqslant 8.0$, with median $\mathrm{S} / \mathrm{N}=19.3$. Each source was measured simultaneously in intensity and polarization. Many sources have also near-simultaneous measurements at 5 and $8 \mathrm{GHz}$, and polarization was detected for 1559 sources in at least one band. Some of the brightest sources were re-observed with $1 \mathrm{mJy}$ polarization sensitivity at $20 \mathrm{GHz}$ (Massardi et al. 2013). Sky areas within $12^{\circ}$ of the nominal centers of the low-foreground QUIET patches contain 531 sources, 86 of which have a $20 \mathrm{GHz}$ polarization measurement (Table 2). Because our cut-out maps may intersect the edge of our survey, we additionally require that each source map must contain a minimum number of pixels $\left(7^{\prime}\right.$

\footnotetext{
26 A smaller improvement is seen for $E E$ than $B B$, because intensity-topolarization leakage tends to primarily induce $E$-mode residuals.
}

Table 2

AT20G Counts Near QUIET Patches

\begin{tabular}{lcccc}
\hline \hline Patch & R.A. & Decl. & $N$ Sources & $N(20 \mathrm{GHz}$ Pol. $)$ \\
\hline CMB-1 & $12: 04: 00$ & $-39: 00: 00$ & 108 & 12 \\
CMB-2 & $05: 12: 00$ & $-39: 00: 00$ & 143 & 26 \\
CMB-3 & $00: 48: 00$ & $-48: 00: 00$ & 130 & 18 \\
CMB-4 & $22: 44: 00$ & $-36: 00: 00$ & 95 & 11 \\
\hline & & Totals & 476 & 67 \\
\hline
\end{tabular}

Note. QUIET low foreground patch centers, the number of sources in the AT20G catalog within $12^{\circ}$ of the center (after cuts for QUIET Q-band sky coverage), and the number of sources with measured $20 \mathrm{GHz}$ polarization.

size), namely 120 for Q-band and 30 for $\mathrm{W}$-band. This costs us minimal statistical power, because the survey sensitivity declines gradually at the patch edges, and those sources are only poorly measured. This cut reduces the number of candidate sources to 476 for Q-band and 480 for W-band. For sources that have AT20G-measured polarization at $20 \mathrm{GHz}, 67$ fall in our Q-band map and 71 fall in our Wband map, due to slight differences in sky coverage.

About $23 \%$ of the sources have a nearby source located within $30^{\prime}$, so to note possible contamination, these sources are flagged in our summary statistics. Since AT20G is $91 \%$ complete above $100 \mathrm{mJy}$ in total intensity (Murphy et al. 2010), we expect that they have captured nearly everything brighter than a few mJy in polarization. QUIET's $1 \sigma$ sensitivity is typically $30-40 \mathrm{mJy}$ per Stokes parameter, so we have not considered source confusion noise beyond this flagging; especially after averaging over polarization directions, it will be small compared to our uncertainty.

\section{METHOD}

\subsection{Estimation of Stokes Parameters}

For a point source of radiation, we model our data as

$$
\boldsymbol{d}=\boldsymbol{F s}+\boldsymbol{n},
$$

where the vector $\boldsymbol{d}$ contains the measured polarization values for pixels near the source, the matrix $s$ is the two-dimensional template for a point source in those pixels, and the vector $s$ contains the polarized flux density and parameters for the template. The vector $\boldsymbol{n}$ denotes instrumental and background noise. The minimum-variance, unbiased source flux density estimate is

$$
\tilde{\boldsymbol{s}}=\left(\boldsymbol{F}^{T} \boldsymbol{N}^{-1} \boldsymbol{F}\right)^{-1} \boldsymbol{F}^{T} \boldsymbol{N}^{-1} d
$$

where $N$ is the instrumental noise covariance.

The template for each source is built from an axisymmetric source profile, sampled at the distances from the source catalog position to the map pixel centers (Schultz \& Huffenberger 2012). The source profile combines the effects of the beam and pixel window function, which are combined in harmonic space, then converted to real space with a Legendre transform. We convert from flux density to temperature units in the source template, using the effective band frequency. As a rule of thumb, a $100 \mathrm{mJy}$ source creates a $24 \mu \mathrm{K}$ peak signal in Qband, and a $29 \mu \mathrm{K}$ peak signal in W-band. As mentioned above, to reduce our sensitivity to large scale modes, we additionally fit and marginalize over a constant planar offset, 
independent for each Stokes parameter, for every source. Thus our model for the vector $s$ includes $\left(S_{Q}, S_{U}, A_{Q}, A_{U}\right)^{T}$, where $\left(S_{Q}, S_{U}\right)$ are the linear polarized flux densities and $\left(A_{Q}, A_{U}\right)$ are the constant offsets.

For point sources, the effective noise matrix $\boldsymbol{N}$ includes contributions from two terms, instrumental noise and $\mathrm{CMB}$ fluctuations. The instrumental contribution dominates, and has a standard deviation of $\sim 20 \mu \mathrm{K}$ for a $7^{\prime}$ Q-band pixel near the center of the map; for a W-band pixel it is typically lower by a factor of 0.6 . Due to the QUIET scanning strategy, the covariance is nearly diagonal, although slightly anti-correlated for adjacent pixels. The second, and smaller, contribution to the covariance is from the $\mathrm{CMB}$, an unavoidable background for these sources. This contribution is evaluated from the CMB power spectrum (Kamionkowski et al. 1997) based on the WMAP7 best-fit spectrum (Larson et al. 2011), accounting for beam window and pixel window functions. The resulting covariance is dense, and has an rms of $\sim 1 \mu K$ for Q-band and $\sim 2 \mu \mathrm{K}$ for $\mathrm{W}$-band; the different amplitudes is due to the different beams. Because the CMB is highly correlated, the Pearson's correlation coefficient is $r \sim 0.8$ or higher for adjacent pixels. Including the $\mathrm{CMB}$ fluctuation in the covariance matrix accounts for $\mathrm{CMB}$ polarization modes which could otherwise masquerade as source flux, and potentially bias the source amplitude.

The polarization estimate $\tilde{\boldsymbol{s}}$ has covariance matrix

$$
\boldsymbol{C}=\left(\boldsymbol{F}^{T} \boldsymbol{N}^{-1} \boldsymbol{F}\right)^{-1}
$$

For each source this is a symmetric $4 \times 4$ matrix, listing every combination of Stokes parameters and offsets. Marginalizing over the offsets amounts to retaining the four entries for the Stokes parameter combinations $Q Q, Q U, U Q$, and $U U$ to build the $2 \times 2$ marginal covariance $\boldsymbol{C}_{m}$. This matrix is nearly diagonal, and the marginal uncertainty for most sources in our sample is less than $100 \mathrm{mJy}$ (see Figure 1), with a median uncertainty per Stokes parameter of $32 \mathrm{mJy}$ for Q-band and $39 \mathrm{mJy}$ for W-band. ${ }^{27}$ We may expect such a wide range in errors because the map sensitivity drops near the edge of each patch. By comparison, in the WMAP 9-year point source catalog, the median error on total intensity is $50 \mathrm{mJy}$ in Q-band and $170 \mathrm{mJy}$ in W-band (Wright et al. 2009), and polarization errors would be bigger by a factor $\sqrt{2}$. For the Planck catalog of compact sources (Planck Collaboration et al. 2014), the median intensity error is $110 \mathrm{mJy}$ at $44 \mathrm{GHz}$ and $60 \mathrm{mJy}$ at $100 \mathrm{GHz}$ after two sky surveys. Errors will improve from the inclusion of three more surveys in the upcoming releases, but for polarization they will also be degraded by a factor $\sqrt{2}$. Thus QUIET's uncertainty per source is competitive with both WMAP and Planck; however, those all-sky experiments have the advantage that they cover 40 times more area.

\footnotetext{
${ }^{27}$ For W-band the noise in temperature units is typically lower than in Q-band by a factor $\sim 0.6$, while the smaller beam concentrates the source signal by a factor of the solid angle ratio $\sim 4$. However, the number of pixels that meaningfully contribute to the estimate is also smaller by the same factor, which increases the error by a factor $\sim \sqrt{4}=2$. The blackbody conversion factor $d B / d T$ to flux density units from temperature is roughly a factor of 5 larger for W-band. So the crude expectation is that W-band errors will be $0.6 / 4 \times 2 \times 5=1.5$ times larger than Q-band errors, while we observe a factor $\sim 1.4$.
}
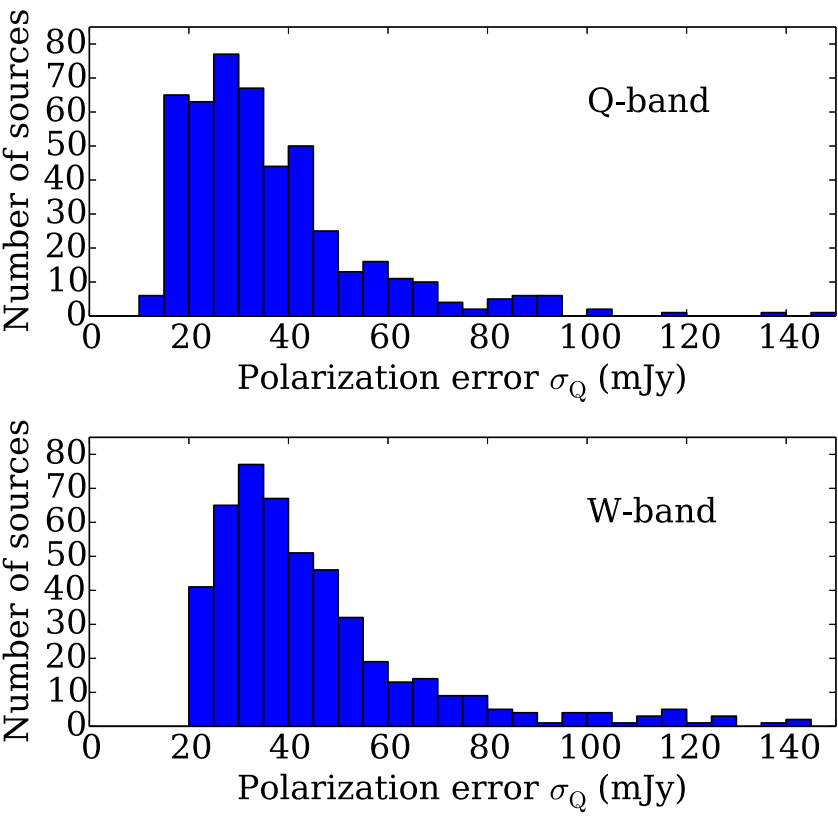

Figure 1. Uncertainty distribution for the Stokes parameter $S_{Q}$, as defined by the square root of the $Q Q$-component of the covariance. Distributions for $\sigma_{U}$ are similar.

For every source we compute

$$
\chi^{2}=\left(S_{Q} S_{U}\right) C_{m}^{-1}\left(S_{Q} S_{U}\right)^{T}
$$

based on the marginalized covariance and the null hypothesis that there is no polarized flux. We then compute the probability-to-exceed (PTE) for the resulting $\chi^{2}$ due to chance alone, $P\left(>\chi^{2}\right)$, where the two Stokes parameters are the two degrees of freedom. Low probability indicates significant flux density. We also compute $\mathrm{S} / \mathrm{N}$ in terms of the equivalent significance in standard deviations for a Gaussian distribution with the same probability,

$$
P\left(>\chi^{2}\right)=1-\operatorname{erf}\left(\frac{\mathrm{S} / \mathrm{N}}{\sqrt{2}}\right),
$$

where erf is the error function. Whenever we discuss the significance of polarized emission, we compute it from the measured Stokes parameters using Gaussian $/ \chi^{2}$ statistics defined in this way.

\subsection{Estimation of Polarized Amplitude}

The amplitude of polarization is positive-definite, requiring non-Gaussian statistics especially when $\mathrm{S} / \mathrm{N}$ is low. Our estimates of the Stokes parameters $S_{Q}$ and $S_{U}$ are unbiased, but computing the polarized flux naively as $S_{P}=\left(S_{Q}^{2}+S_{U}^{2}\right)^{1 / 2}$ is biased by the noise. This bias is treated elsewhere in the literature: Simmons \& Stewart (1985) account for the biases when Stokes parameters are uncorrelated and have the same errors, using properties of the Rice distribution; Quinn (2012) has a detailed treatment of Bayesian estimators, again for equal errors; Plaszczynski et al. (2014) develop an analytic, approximate distribution for the general case of correlated errors; Vidal et al. (2014) treat a case with an already-known polarization angle; Montier et al. (2015a, Appendices A-D) examine for the distributions in the case of correlated errors, 
writing the marginal distribution for polarization as a series expansion; and Montier et al. (2015b) compare several estimation methods. Before the Montier et al. (2015a) method was made public, we had developed an alternative approach, described below, which retains the full correlated information via a Monte Carlo method. (Although in our case Stokes parameters are approximately uncorrelated.)

For each source, we seek the posterior probability $(\mathcal{P})$ for the true Stokes parameters $\left(S_{Q}, S_{U}\right)$, the polarized flux $\left(S_{p}\right)$, and polarization angle $(\alpha)$, all conditioned on our observed Stokes parameters. The covariance we computed gives the likelihood $(\mathcal{L})$ of an observation based on the true value. Using Bayes theorem and a uniform prior, we write the posterior as

$$
\mathcal{P}\left(S_{Q}, S_{U} \mid S_{Q}^{\text {obs }}, S_{U}^{\text {obs }}\right) \propto \mathcal{L}\left(S_{Q}^{\text {obs }}, S_{U}^{\text {obs }} \mid S_{Q}, S_{U}\right),
$$

which is a $2 \mathrm{D}$ Gaussian distribution with mean and covariance given by the measurement. To compute the distribution of $S_{P}$ (and $\alpha$ ), we generate samples of the Stokes parameters from this Gaussian distribution. If the errors on Stokes parameters are not Gaussian, our method can be generalized to whatever distribution is appropriate. We then multiply with the Jacobian to transform the sampled distribution while conserving probability,

$$
\mathcal{P}\left(S_{Q}, S_{U}\right)=\mathcal{P}\left(S_{P}, 2 \alpha\right)\left|\frac{\partial\left(S_{Q}, S_{U}\right)}{\partial\left(S_{P}, 2 \alpha\right)}\right|=\mathcal{P}\left(S_{P}, 2 \alpha\right) S_{p} .
$$

Finally, we marginalize the right-hand distribution to produce the 1D posterior for the polarized flux $\mathcal{P}\left(S_{P} \mid S_{Q}^{\text {obs }}, S_{U}^{\text {obs }}\right)$. This simply amounts to binning the samples, dividing the bin value by $S_{P}$, then normalizing. We construct the distribution for the angle in a similar way.

We use $10^{7}$ samples for each source, which probes the shape of the likelihood sufficiently in only a few seconds per source. In Figure 2 we show that this method reproduces the Rice distribution behavior from Simmons \& Stewart (1985, Figure 2). As there, for sources with measured polarized emission less than $~ 1.41$ times the error per Stokes parameter, the maximum likelihood value for the true polarization is zero, and the measured polarization is likely the result of noise alone. For sources with larger measured polarization, the maximum likelihood value exceeds zero, and at high $\mathrm{S} / \mathrm{N}$, the maximum likelihood estimate approaches the true value.

When the $\mathrm{S} / \mathrm{N}$ for a given source is high, the likelihood peaks well away from zero polarized flux. Like Vaillancourt (2006), we base confidence intervals for these cases on the integrated probability, and report the median $68 \%$ interval (from the 16th to 84th percentile) of the posterior, which contains the maximum posterior point. When the $\mathrm{S} / \mathrm{N}$ for a source is low, the polarization likelihood peaks near zero polarized flux, and the maximum posterior point is typically closer to zero than the median $68 \%$ interval. In this paper, we report a $95 \%$ upper limit for any source with $\mathrm{S} / \mathrm{N}<2$ (computed from Equation (6)).

\section{RESULTS}

The two brightest objects in our fields, Centaurus A and Pictor A, have structures that are extended compared to our beams. We report results for these first, followed by the larger population of fainter objects, which we treat as point sources.
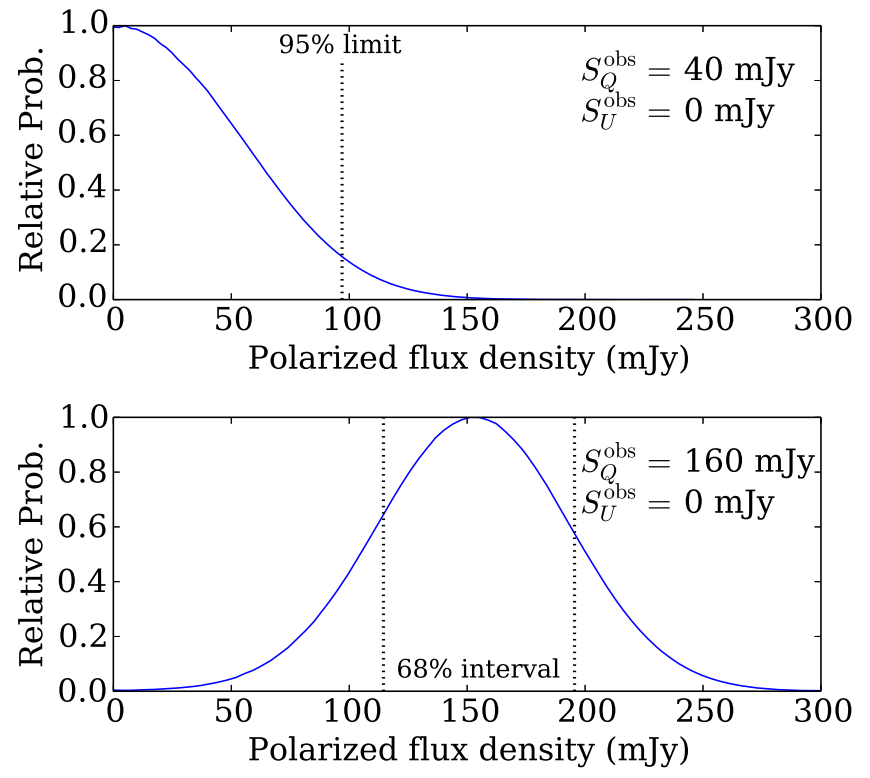

Figure 2. Example posteriors for polarized flux density measurements. Both have diagonal covariances with $\sigma_{Q}=\sigma_{U}=40 \mathrm{mJy}$. Top: for low $\mathrm{S} / \mathrm{N}$, in this case a $40 \mathrm{mJy}$ observation, the maximum probability point for the true polarization is near zero, and we accordingly quote only a $95 \%$ upper limit. Bottom: for higher $\mathrm{S} / \mathrm{N}$, such as a $160 \mathrm{mJy}$ observation, the maximum probability for the true polarization occurs near the observed value. In cases like these we quote the $68 \%$ interval.

\subsection{Extended Sources}

The brightest object in our fields is the radio galaxy Centaurus A (Cen A). In radio images this object is nearly $10^{\circ}$ across, and has several major components (Junkes et al. 1993), including the Northern and Southern Giant Outer Lobes, the Northern Middle Lobe (30' north of the core), the inner lobes, and the nuclear region. With our angular resolution, we can only resolve the outer and middle lobes. Also, Cen A lies in the outskirts of our patch CMB-1, in a region with fewer observations, less cross-linking, and higher noise than the bulk of our survey. It lies outside the normal processing mask for our maximum likelihood pipeline. Nonetheless, Cen A is so bright that a naive (binned) map of our time-ordered data is sufficient to obtain useful results. For QUIET in polarization, this naive map differs from the full solution only on large scales. But since we use the binned map we limit ourselves here to a qualitative discussion.

This map is shown in Figure 3. The images are centered on the peak of the galaxy's light from a 2 MASS $\mathrm{K}_{\mathrm{S}}$-band image (Skrutskie et al. 2006). Polarized emission is brighter in Qband than in W-band. The noise is higher to the left, a gradient expected from the position of the source at the outskirts of the QUIET field. In Q-band, polarized emission is apparent in the outer lobes even $2^{\circ}$ away from the galaxy. The lobes have the same slight " $\mathrm{S}$ " shape as seen in total intensity (in WMAP, Planck, and multiple radio observations) and in radio polarization. The peak emission is offset from the galaxy. In Q-band, the peak emission is centered on two spots on either side of the galaxy, at $(\Delta l \cos b, \Delta b) \sim\left(-5^{\prime}, 30^{\prime}\right)$ (near the Northern Middle Lobe) and another spot at $\sim\left(0^{\prime}, 15^{\prime}\right)$. Comparing WMAP and Planck images of the lobe, the peaks of polarized emission lie nearer to the galaxy than the peaks of total intensity. In W-band, the brightest emission is nearer to 
QUIET Stokes $Q$
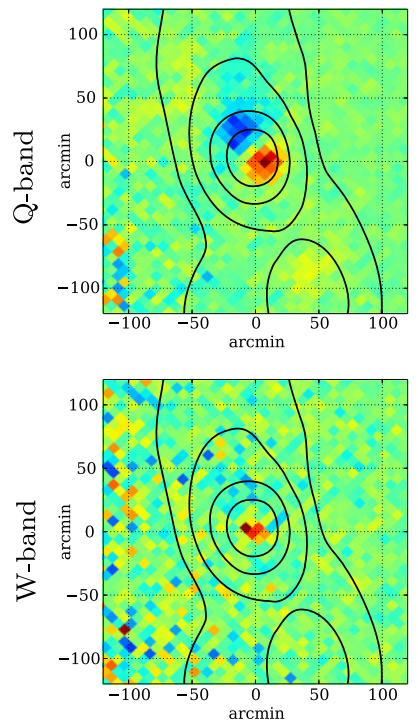

QUIET Stokes $U$
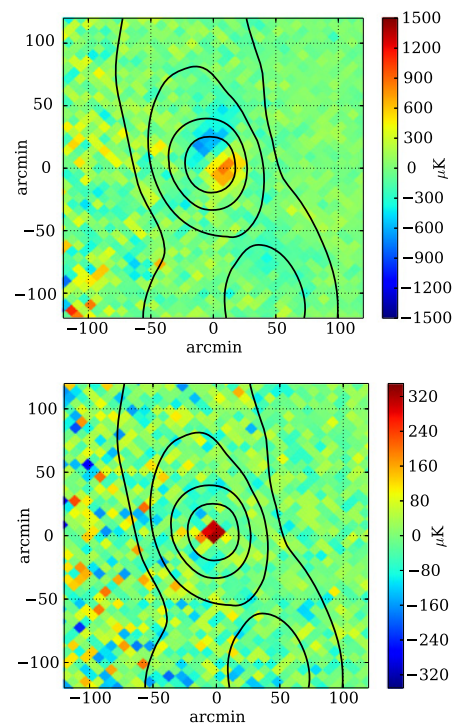

QUIET Polarization
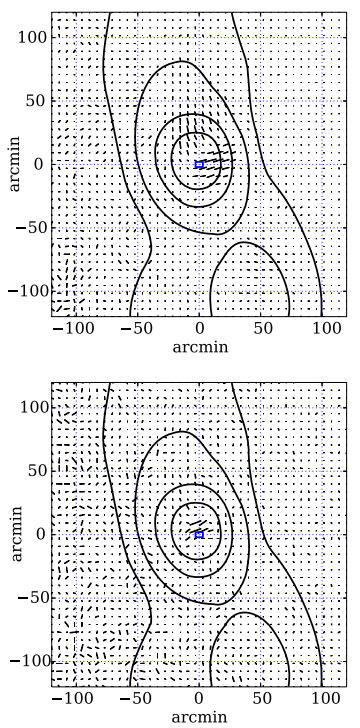

WMAP T
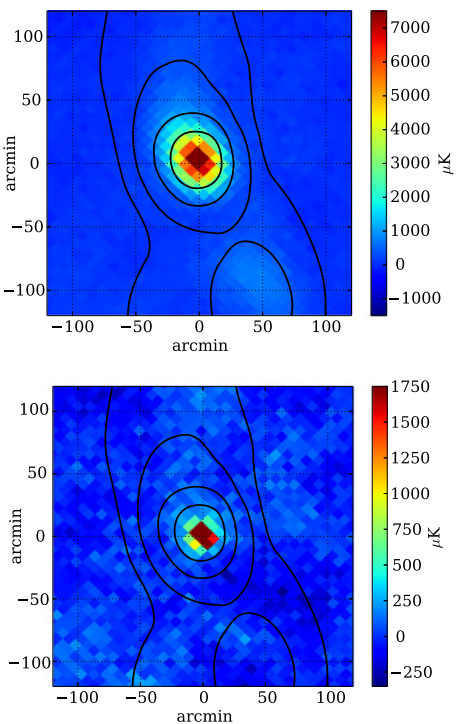

Figure 3. Radio galaxy Cen A $(l=309: 516, b=19: 417)$, showing Stokes $Q$ and $U$, polarization vectors, and total intensity from WMAP (Bennett et al. 2013), for Qband $\left(43 \mathrm{GHz}\right.$, above) and $\mathrm{W}$-band $\left(95 \mathrm{GHz}\right.$, below). Images are $4^{\circ}$ on a side, and show naively binned maps in gnomonic projection; Galactic longitude increases to the left. For comparison, we plot contours from the Haslam et al. (1982) radio map at $408 \mathrm{MHz}$, indicating radio brightness temperatures $T_{B}=70,140,280,420 \mathrm{~K}$ (from the outside in). The small blue box in the vector image, the central $6 \times 4$ sq. arcmin, spans the optical/NIR extent of the galaxy's disk, and by comparison emphasizes the large size of the radio lobes. Note that the most intense polarization is not centered on the galaxy.

the galaxy than in Q-band, and the brightest emission is in fact concentrated in one spot, at $(\Delta l \cos b, \Delta b) \sim\left(0^{\prime}, 5^{\prime}\right)$.

Note that the peaks of the polarized emission in Q-band and W-band differ in location and intensity, and the polarization direction varies strongly as a function of position. This is instructive as we later consider the population of unresolved sources. For the same reason, our measurements of point-like sources below cannot easily probe rotation measure and magnetic fields; for unresolved sources, we cannot be sure that we are always probing regions with the same intrinsic polarization direction. This may be why, for our larger set of point-like sources, the polarization intensities and directions show little correlation between the two frequencies.

Another bright source, radio galaxy Pictor A (Pict A), lies in our CMB-2 field. This object also has multiple components; the central nucleus and both radio lobes appear as separate entries in the AT20G catalog, ${ }^{28}$ with a total lobe-to-lobe separation of about $8^{\prime}$. This separation is smaller than the FWHM of our beams, so we cannot separately resolve the components. Figure 4 shows that our data are noisy, but Pict A is nonetheless detected in polarization at $\mathrm{S} / \mathrm{N}=7.75$. At $20 \mathrm{GHz}$, Murphy et al. (2010) find that the nucleus $(6320 \pm 110 \mathrm{mJy})$ is much brighter than the western lobe $(1464 \pm 55 \mathrm{mJy})$ in total intensity. However, they detect no significant polarization from the nucleus, while the western lobe has a large polarized flux density $(423 \pm 5 \mathrm{mJy})$. The western lobe also has stronger polarization at lower frequencies $<5 \mathrm{GHz}$ (Perley et al. 1997). Using our standard photometry method, as described in Section 3.1, our observations also yield the largest polarization toward the western lobe, with a maximum likelihood polarization of $S_{P}=205_{-21}^{+31} \mathrm{mJy}$ at Qband and $S_{P}=89_{-33}^{+33} \mathrm{mJy}$ at W-band.

\footnotetext{
${ }^{28}$ Western lobe: AT20G J051926-454554; nucleus: AT20G J051949-454643; eastern lobe: AT20G J052006-454745.
}

Q
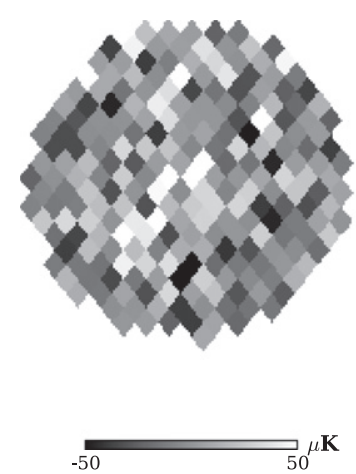

$\mathrm{U}$
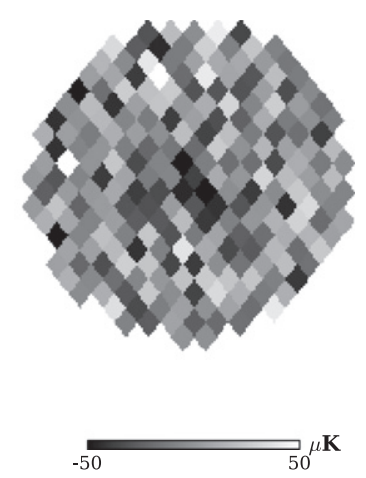

Figure 4. The data are noisy, but Pict A, at the center of this Q-band image, is nonetheless detected at $\mathrm{S} / \mathrm{N}=7.75$, with a positive Stokes $Q$ and negative Stokes $U$. The unmasked region has a $120^{\prime}$ diameter, and the beam FHWM is 27.3 .

However, because of our beam sizes and the close proximity of the components, our standard photometry method is suboptimal, as it does not account for overlapping emission. Therefore, for Pict A we also compute the flux density by integrating over top-hat apertures of increasing sizes, which provides some notion of the total polarization, even though we lack the resolution to examine the three components of Pict A individually. For Q-band, the polarization signal increases as the aperture expands up to $\sim 30^{\prime}$ in radius, and is then constant within the errors. For that aperture, we find $\left(S_{Q}, S_{U}\right)=(105 \pm 40,-146 \pm 39) \mathrm{mJy}$, consistent to our standard fit, but with larger error bars. ${ }^{29}$ In the same $30^{\prime}$ aperture for W-band, we find $\left(S_{Q}, S_{U}\right)=$ $(104 \pm 77,-249 \pm 77) \mathrm{mJy}$, but the signal is not constant as

\footnotetext{
29 Aperture photometry is less restrictive than the fitted template from Section 3 , and so less precise.
} 
Table 3

S/N > 2.7 Detections, Q-band

\begin{tabular}{|c|c|c|c|c|c|c|c|c|}
\hline ID & R.A. & Decl. & $S_{Q}^{\mathrm{Gal}}(\mathrm{mJy})$ & $S_{U}^{\mathrm{Gal}}(\mathrm{mJy})$ & $S_{P, \mathrm{ML}}(\mathrm{mJy})$ & $\alpha_{\mathrm{IAU}}^{\mathrm{Cel}}\left({ }^{\circ}\right)$ & $\operatorname{Pr}\left(>\chi^{2}\right)$ & $\mathrm{S} / \mathrm{N}$ \\
\hline AT20GJ010645-403419 & $1: 06: 45.11$ & $-40: 34: 19.5$ & $16 \pm 42$ & $160 \pm 41$ & $154_{-40}^{+43}$ & $304_{-6}^{+10}$ & 0.00052 & 3.47 \\
\hline AT20GJ042840-375619 & $4: 28: 40.37$ & $-37: 56: 19.2$ & $102 \pm 28$ & $-8 \pm 28$ & $98_{-29}^{+29}$ & $269_{-8}^{+8}$ & 0.0014 & 3.19 \\
\hline AT20GJ050838-330853 & $5: 08: 38.05$ & $-33: 08: 53.5$ & $-86 \pm 23$ & $4 \pm 23$ & $84_{-22}^{+24}$ & $170_{-6}^{+8}$ & 0.0008 & 3.35 \\
\hline AT20GJ051926-454554, & $5: 19: 26.34$ & $-45: 45: 54.6$ & $153 \pm 26$ & $-143 \pm 26$ & $207_{-26}^{+28}$ & $287_{-3}^{+4}$ & $9.5 \times 10^{-15}$ & 7.75 \\
\hline AT20GJ053757-461430 & $5: 37: 57.60$ & $-46: 14: 30.3$ & $29 \pm 30$ & $-90 \pm 30$ & $91_{-32}^{+30}$ & $298_{-8}^{+10}$ & 0.0063 & 2.73 \\
\hline AT20GJ111301-354947 & $11: 13: 01.51$ & $-35: 49: 47.5$ & $43 \pm 36$ & $-108 \pm 36$ & $112_{-39}^{+36}$ & $238_{-8}^{+10}$ & 0.0065 & 2.72 \\
\hline AT20GJ113855-465342 ${ }^{\mathrm{a}}$ & $11: 38: 55.60$ & $-46: 53: 42.6$ & $91 \pm 36$ & $-98 \pm 36$ & $127_{-35}^{+38}$ & $219_{-8}^{+8}$ & 0.0011 & 3.25 \\
\hline AT20GJ123045-312123 & $12: 30: 45.02$ & $-31: 21: 23.1$ & $109 \pm 30$ & $-26 \pm 31$ & $109_{-31}^{+31}$ & $193_{-6}^{+10}$ & 0.001 & 3.29 \\
\hline AT20GJ224326-393352 & $22: 43: 26.04$ & $-39: 33: 52.6$ & $-100 \pm 33$ & $46 \pm 31$ & $104_{-33}^{+36}$ & $357_{-6}^{+10}$ & 0.0034 & 2.93 \\
\hline
\end{tabular}

Note. AT20G catalog locations that show $43 \mathrm{GHz}$ polarized flux density at a statistical significance equivalent to S/N > 2.7 in QUIET Q-band data. Stokes parameters use Galactic coordinates and CMB convention, while the polarization angle uses Celestial coordinates and IAU convention (see discussion at end of Section 1). Only the source AT20GJ042840-375619 appears in both Tables 3 and 4.

a Another AT20G source lies within 30'.

${ }^{\mathrm{b}}$ Component of Pict A.

Table 4

S/N > 2.7 Detections, W-band

\begin{tabular}{|c|c|c|c|c|c|c|c|c|}
\hline ID & R.A. & Decl. & $S_{Q}^{\mathrm{Gal}}(\mathrm{mJy})$ & $S_{U}^{\mathrm{Gal}}(\mathrm{mJy})$ & $S_{P, \mathrm{ML}}(\mathrm{mJy})$ & $\alpha_{\mathrm{IAU}}^{\mathrm{Cel}}\left({ }^{\circ}\right)$ & $\operatorname{Pr}\left(>\chi^{2}\right)$ & $\mathrm{S} / \mathrm{N}$ \\
\hline AT20GJ000601-423439 & 0:06:01.95 & $-42: 34: 39.8$ & $135 \pm 45$ & $44 \pm 45$ & $135_{-46}^{+46}$ & $26_{-8}^{+10}$ & 0.0067 & 2.71 \\
\hline AT20GJ005645-445102 & $0: 56: 45.80$ & $-44: 51: 02.4$ & $83 \pm 26$ & $-37 \pm 26$ & $86_{-26}^{+28}$ & $9_{-6}^{+10}$ & 0.0026 & 3.01 \\
\hline AT20GJ042840-375619 & $4: 28: 40.37$ & $-37: 56: 19.2$ & $248 \pm 37$ & $5 \pm 37$ & $246_{-38}^{+38}$ & $266_{-4}^{+5}$ & $2.8 \times 10^{-10}$ & 6.31 \\
\hline AT20GJ052257-362730 & $5: 22: 57.94$ & $-36: 27: 30.4$ & $90 \pm 25$ & $-10 \pm 25$ & $88_{-25}^{+25}$ & $262_{-6}^{+10}$ & 0.0015 & 3.17 \\
\hline AT20GJ053850-440508 & $5: 38: 50.35$ & $-44: 05: 08.7$ & $103 \pm 30$ & $73 \pm 30$ & $122_{-29}^{+32}$ & $244_{-6}^{+8}$ & 0.00014 & 3.81 \\
\hline AT20GJ054922-405107 & $5: 49: 22.79$ & $-40: 51: 06.9$ & $86 \pm 33$ & $-76 \pm 33$ & $107_{-31}^{+37}$ & $279_{-6}^{+10}$ & 0.0027 & 3.00 \\
\hline
\end{tabular}

Note. Same as Table 3, but for $95 \mathrm{GHz}$ polarized flux density in QUIET W-band data. Only the source AT20GJ042840-375619 appears in both Tables 3 and 4. ${ }^{a}$ Another AT20G source lies within $30^{\prime}$.

the aperture is increased further, so we are unable to fit a constant background offset. This is due to large scale features in the map, and so this W-band aperture photometry may be unreliable. To avoid multiple counting in our summary statistics below, we either include only the western lobe of Pict A, or exclude it altogether.

\subsection{Point Sources}

For the remainder of the AT20G sources, which are selected at $20 \mathrm{GHz}$, we measure the values of the Stokes parameters at 43 and $95 \mathrm{GHz}$. Depending on detection significance, we provide a measurement or an upper limit on the polarized flux density, as described above. Further, for some of the AT20G sources that exceed $\mathrm{S} / \mathrm{N}>2.0$ in our measurements, an additional AT20G source lies within $30^{\prime}$. However, except for the components of Pict A, all of those neighboring sources have $\mathrm{S} / \mathrm{N}<2.0$ significance in our measurements, limiting the potential for contamination.

We find a handful of point-like sources producing polarized flux density at high $\mathrm{S} / \mathrm{N}$, as listed in Tables 3 and 4 . We adopt a threshold of $\mathrm{S} / \mathrm{N}>2.7$ for these tables, which is a compromise that keeps the table short and limits the number of spurious detections. At that level of significance or greater, we find 11 independent sources in Q-band and 7 sources in W-band. Only AT20GJ042840-375619 (the well-known quasar PKS 0426-380) exceeds the significance cut in both Q- and $\mathrm{W}$-bands. Accounting for the total number of AT20G sources, noise alone should account for $3.3 \pm 1.8$ detections of polarized emission in each table, based on Poisson statistics. At $\mathrm{S} / \mathrm{N}>3.0$ or greater, only $1.3 \pm 1.1$ detections should be spurious, while we find seven in Q-band and six in W-band. We therefore conclude that the greater part of the listed sources record genuine polarized emission. Figure 5 shows the brightest sources for W-band.

For a sensitivity cut of $\mathrm{S} / \mathrm{N}>3$, it is interesting to note that we find similar numbers of sources in the more sensitive Qband at $43 \mathrm{GHz}$ and the slightly less sensitive W-band at $95 \mathrm{GHz}$ (see Figure 1). If these sources were equally bright in both bands we would tend to see fewer in W-band. Of course, we cannot draw strong conclusions based on this small number of sources, but this observation may suggest that source polarization is fairly flat over this frequency range. WMAP found that the mean spectral index in intensity is also nearly flat (and slightly negative): $S \propto \nu^{\alpha_{\mathrm{SED}}}$ with mean $\alpha_{\mathrm{SED}} \pm \Delta \alpha_{\mathrm{SED}}=-0.09 \pm 0.28$, where the range indicates source-to-source scatter (Wright et al. 2009). 
Q

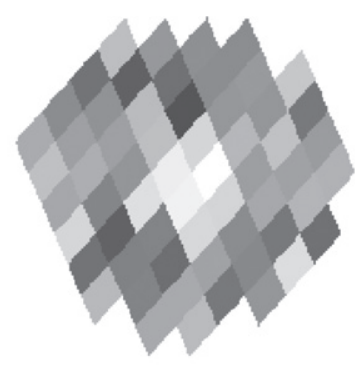

$-50$

Q

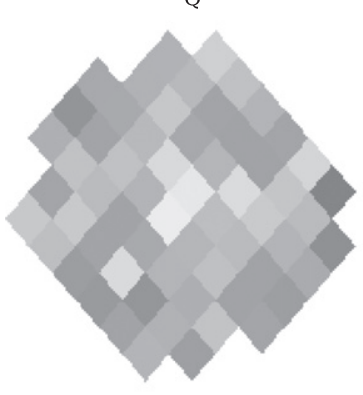

$-50$
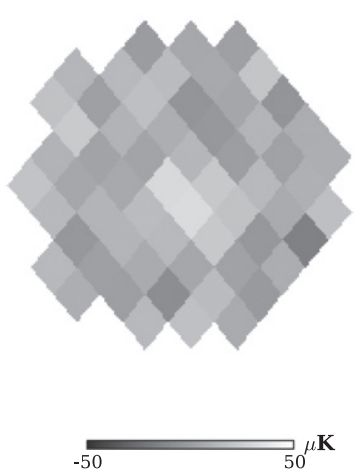

$\mathrm{U}$
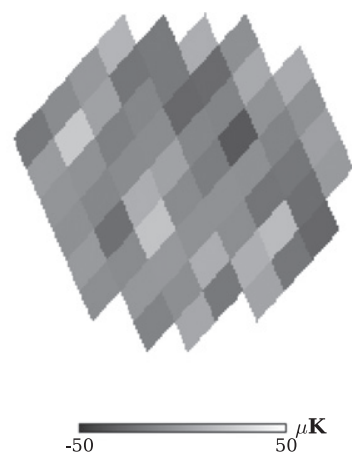

$\mathrm{U}$
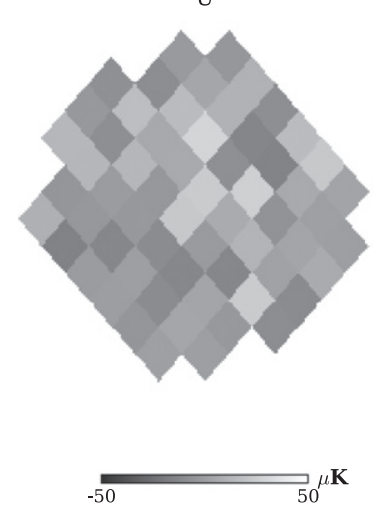

$\mathrm{U}$
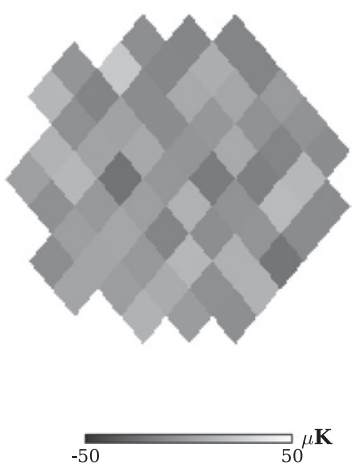

Figure 5. The three sources detected in W-band at greatest significance (from top): AT20GJ042840-375619, AT20GJ053850-440508, and AT20GJ052257362730. The unmasked regions have $60^{\prime}$ diameters, and the beam FWHM is $12 \cdot 8$.

In Figure 6, we show our polarization measurements of sources compared to the $20 \mathrm{GHz}$ total intensity on which they were selected. For each band, we separate sources observed with $\mathrm{S} / \mathrm{N}>2.7$ from the lower significance sources to make the plots clearer. For higher $\mathrm{S} / \mathrm{N}$ sources, we show the maximum likelihood point and the 68\% interval, as illustrated in Figure 2. Conversely, for lower $\mathrm{S} / \mathrm{N}$ sources, we only plot the maximum likelihood point and a 95\% upper limit. The sensitivity of our upper limit depends on the data and the errors at that position on the map. Half of the sources have polarizations below $90 \mathrm{mJy}$ in Q-band and below $106 \mathrm{mJy}$ in W-band, at 95\% confidence.

No trend is immediately clear. Our strongest detections tend to come from sources with $20 \mathrm{GHz} S>1 \mathrm{Jy}$, but among those
19 sources, several yield only upper limits in our data. This may indicate diversity in the higher-frequency polarization properties of the sources selected from the $20 \mathrm{GHz}$ catalog. Simple models, which fix both the frequency scaling $\left(\alpha_{\mathrm{SED}}\right)$ and the polarization fraction (at a few percent), will not reproduce this result.

\subsection{Validation and Robustness}

We now consider the robustness of these results by means of null maps and simple simulations. The null map used in the following was produced in the course of the CMB power spectrum analysis for the Q-band, in which the data were split into the first and second halves of the first observing season, comprising 232 days from 2008 October-2009 June. Subtracting (rather than adding) these half-datasets during mapmaking produces the null map. Ideally, any time-independent signal should cancel in this map, leaving only residual noise and, possibly, variable sources.

We run the null map cutouts through the same pipeline as the main analysis, and show in Figure 7 the resulting distributions of source significances. Here we see that the Q-band and Wband maps show an excess of high-significance polarization measurements compared to the expectation from noise alone. By contrast, for the source positions in the Q-band null map, we find fewer indications of additional polarized flux beyond noise: at $\mathrm{S} / \mathrm{N}>3$ we expect $1.3 \pm 1.1$ spurious detections, and find 2. These consistent values for the null map strengthen our confidence in the covariance matrices.

Both of the $\mathrm{S} / \mathrm{N} \geqslant 3$ detections seen in the null map could be spurious and yet still consistent with noise. However, one source, AT20GJ111301-354947, which has $\mathrm{S} / \mathrm{N}=3.0$ in the null map, has $S / \mathrm{N}=2.7$ in the standard summed map and also appears in Table 3. The Stokes parameters are nearly the same in the null map and the standard map. This may be explained by source variability: if a source produces emission during just one half of the observing season, it does not matter if the quiescent half of the data is added (standard map) or subtracted (null map). The other significant source in the null map is AT20GJ053850-440508, which has $\mathrm{S} / \mathrm{N}=3.4$ in the null map and $\mathrm{S} / \mathrm{N}=0.55$ in the standard map. In intensity, this source is very bright and highly variable, varying during 2009 September-2010 March from $6.141 \pm 0.089 \mathrm{Jy}$ to $14.814 \pm$ $0.194 \mathrm{Jy}$ at $39.8 \mathrm{GHz}$ according to (Massardi et al. 2011a), although that work does not include a measurement of polarization. Although it is suspicious that this is one of the brightest sources in our region, it is hard to reconcile our large polarized detection in the null map with the lack of detection in the standard map, unless it is a noise fluctuation. Given the number of source positions in our fields, noise alone could mimic a $\mathrm{S} / \mathrm{N}>3.4$ source with a chance of one in four.

Our next tests of robustness use synthetic maps. Specifically, we simulate maps in which every source is assigned zero flux density, but has the same noise and covariance properties given by the maximum likelihood pipeline. The measured fluxes from this simulation are statistically consistent with noise. Finally, we ran two simulations including simulated sources with either $100 \mathrm{mJy}$ or $1 \mathrm{Jy}$ amplitudes and randomized polarization directions, and find that our recovered Stokes parameters are unbiased. 

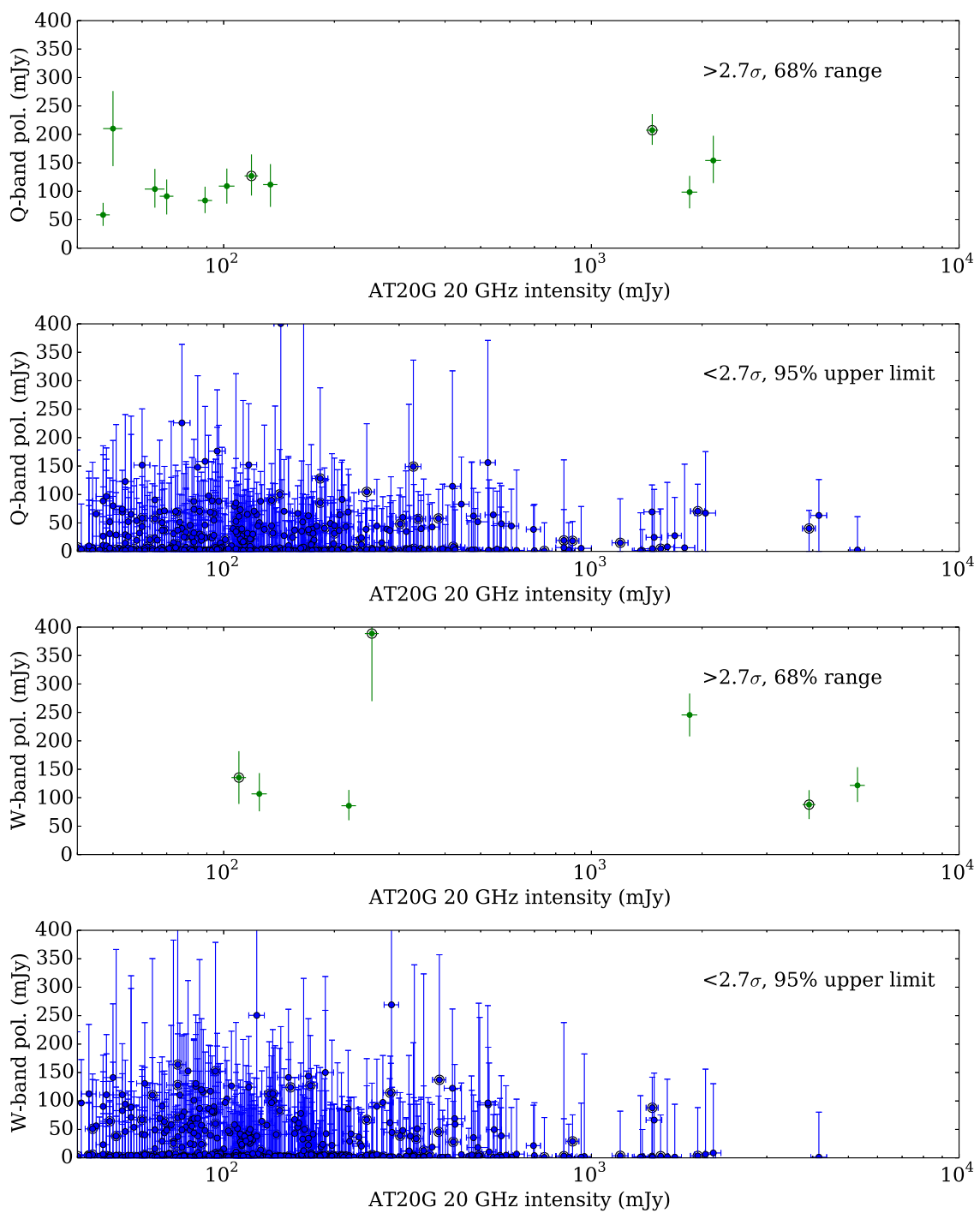

Figure 6. Polarized flux density measured with QUIET vs. the total intensity at $20 \mathrm{GHz}$, where the sources are selected from the AT20G catalog. The top two plots are Q-band ( $43 \mathrm{GHz}$, see Table 3) and the bottom two plots are W-band (95 GHz, see Table 4). The higher-significance subsets, with S/N > 2.7, show 68\% confidence intervals around the maximum likelihood point, and are plotted separately from the upper limits. In the upper-limit plots, each vertical error bar shows the $95 \%$ confidence interval, and connects to zero through the point that mark the maximum likelihood value. Sources marked with a ring have another AT20G source within $30^{\prime}$.

\subsection{Inter-frequency Comparisons}

We now compare the polarization measurements between our bands and also to other measurements of the same sources in the literature.

\subsection{1. $43 \mathrm{GHz}$ Polarization Versus $95 \mathrm{GHz}$ Polarization}

We expect that bright sources will tend to be bright in polarization in both bands, because they are intrinsically luminous, nearby, or both. However, the low $\mathrm{S} / \mathrm{N}$ of our measurements complicate our ability to see this correlation between bands in all but the very brightest sources.

Overall, there are 460 sources in the overlapping sky area covered by both frequency bands. Imposing a S/N > 1.0 cut in both bands, from Poisson statistics and in the absence of any genuine source flux, we expect $47.3 \pm 6.9$ detections to exceed the threshold due to noise fluctuations alone, but instead find 60 detections, indicating some genuine contribution to the polarized emission. Unfortunately, we cannot tell which of these 60 show genuine emission and which make the cut due to noise. At $\mathrm{S} / \mathrm{N}>1.5$ in both bands, we expect $8.3 \pm 2.9$ detections (from noise) but find 17. At $\mathrm{S} / \mathrm{N}>2.0$ in both bands, we begin to run out of objects, expecting $1.2 \pm 1.1$ detections but finding 3 .

Because of the non-Gaussian statistics of the polarization amplitude, we must be very cautious interpreting our measurements. For example, for $\mathrm{S} / \mathrm{N}>1$, the polarized flux density shows a positive correlation between Q- and W-band, with Pearson's $r=0.46$. However, our tests with a synthetic catalog (of spurious detections from noise fluctuations) indicate that this effect is mostly statistical. It results due to scanning depth: the noise levels have a similar spatial variations as a function of position for Q-band and W-band, so the ability for a particular location to have large upward fluctuations in polarization amplitude is correlated between the bands. With a higher $\mathrm{S} / \mathrm{N}$ cut, $\mathrm{S} / \mathrm{N}>1.5$, the correlation coefficient drops to $r=0.11$. Similarly, among the full set of 460 sources, 252 have a smaller maximum likelihood polarization amplitude in Q-band than in 

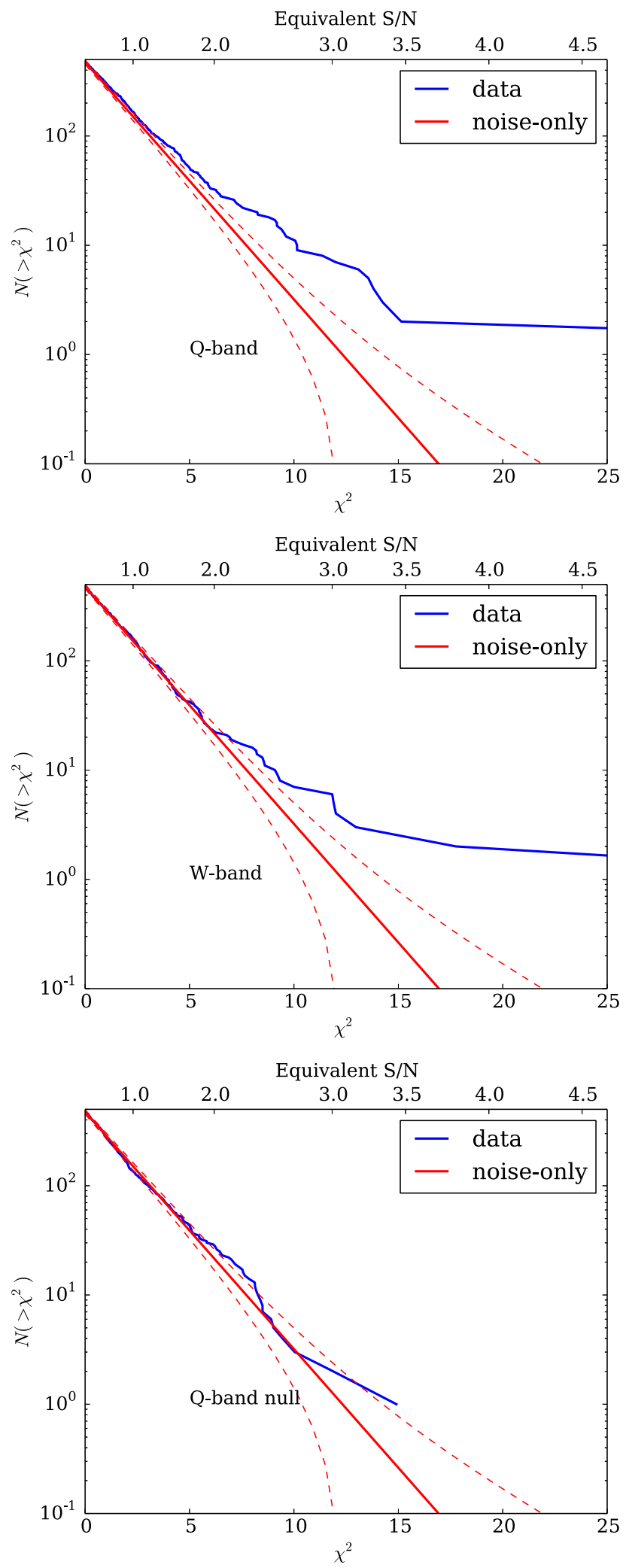

Figure 7. Cumulative number of polarized detections vs. $\chi^{2}$ (in blue), also showing the equivalent significance ( $\mathrm{S} / \mathrm{N}$ from Equation (6)), compared to the expected distribution of spurious detections from noise alone (in red). The red line is the expected distribution from a cumulative $\chi^{2}$-distribution under the null hypothesis that source positions show no excess polarized flux density. The dashed $1 \sigma$ error bars are given by Poisson statistics. The null hypothesis is a good fit for the null map, but a poor fit for the standard maps; each shows excess high-significance objects, evidence of polarized emission.
W-band, and among the 60 sources with $\mathrm{S} / \mathrm{N}>1,36$ have a smaller amplitude in Q-band. This, too, seems to be a statistical effect, and is not due to the source spectral energy distribution (SED), as our tests based on noise-only synthetic catalogs exceed this threshold in similar or greater numbers. This bias toward W-band can be caused by (1) the positive-definite probability distribution for polarization amplitude and (2) the larger error bars in W-band which cause larger excursions (which skew positive) from the true flux density.

Another approach we advocate is to compare the Stokes parameters individually between the bands. Because the errors in the Stokes parameters are Gaussian distributed, they are simpler statistics, and each set is an independent probe. This should be useful for future experiments with larger numbers of high $\mathrm{S} / \mathrm{N}$ sources. In that case, any trend should be the same for each Stokes parameter, due to the random orientation of objects on the sky. Here however, since we only measure a few sources well, we are limited by our significant scatter.

In Figure 8 we plot the Stokes parameters in one band versus the other band. Here the correlation among $\mathrm{S} / \mathrm{N}>1$ sources (in both bands) is weak, with $r=0.020$ for Stokes $Q$ at $43 \mathrm{GHz}$ compared to Stokes $Q$ at $95 \mathrm{GHz}$, and $r=-0.12$ for Stokes $U$ similarly between the bands. We compared to simulations (not shown in the plot) of sources that either have intrinsically correlated Stokes parameters between the bands $\left(r_{\text {input }}=1\right)$, or no correlation $\left(r_{\text {input }}=0\right)$. The sources in these simulations have zero-mean, Gaussian-distributed Stokes parameters with variance roughly matched to our data. Due to noise fluctuations, the measured correlation of the $r_{\text {input }}=1$ simulations is $r=0.4-0.7$, much larger than the correlation we observe.

To explore further the slope of any correlation, we fit a linear model to the data, accounting for the error bars in both directions. Based on the measurement error, we write down $\chi_{\mathrm{QW}}^{2}$ as a function of the intercept and angle (slope) of the fitting line (Press et al. 1992, Equation (15.3.2)), then minimize to find the best fit correlation. To understand the probability of this model we construct a Gaussian distribution proportional to $\exp \left(-\chi_{\mathrm{QW}}^{2} / 2\right)$. To find the probability of the angle we marginalize over the intercept. This is plotted to the right of Figure 8. We expect a positive correlation, and both Stokes parameter correlations peak in the positive quadrant, but the distributions for the angle are broad, and differ. In our perfectly-correlated simulations, the probability peaks sharply on the input slope, with a FWHM of the distribution of 0.16 radians. In the real data, the FHWMs of the angle distributions are larger, 0.38 radians for Stokes $Q$ and 0.27 radians for Stokes $U$. Similar values are seen in the $r_{\text {input }}=0$ simulations.

The $\chi^{2}$-statistic in this approach addresses measurement error but not the intrinsic scatter in our sources, and to this we attribute the marginally significant difference between the directions preferred by Stokes $Q$ and Stokes $U$. Thus we find little evidence that the individual Stokes parameters correlate between the bands.

This we might expect if - as in Cen A-different physical parts of the AGN are bright in polarization at different frequencies. If true generally, it means that predicting accurately the polarization angle of an unresolved source, based on measurements at other frequencies, will not be straightforward without a detailed physical model per object. 

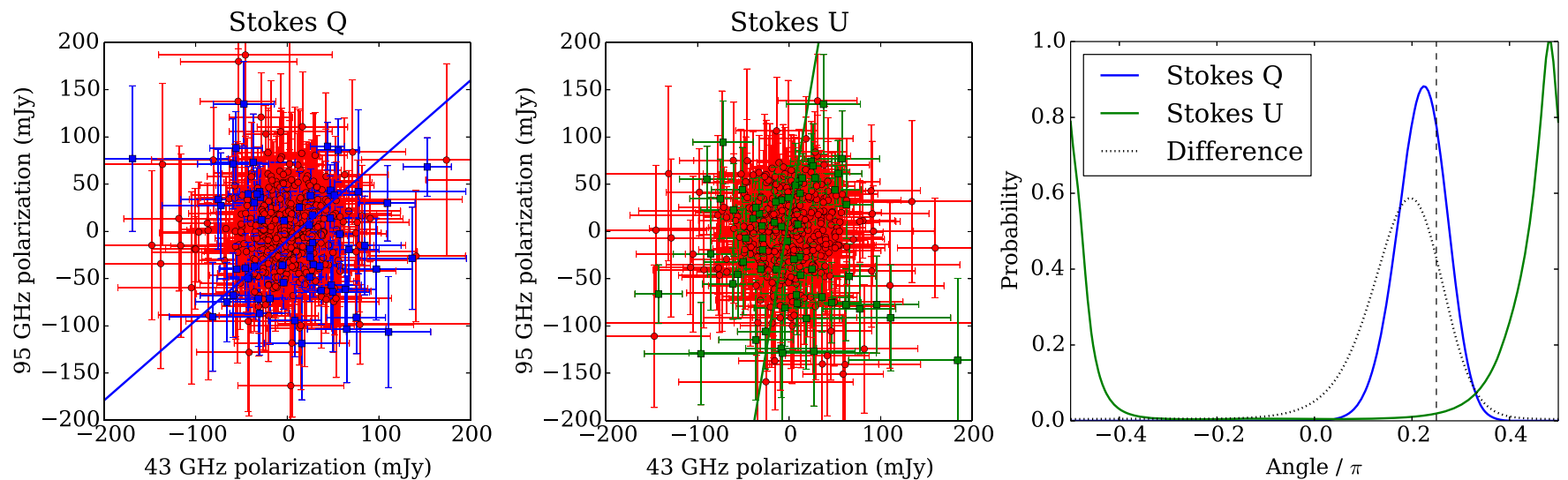

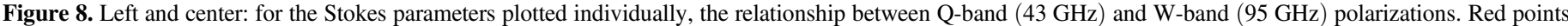

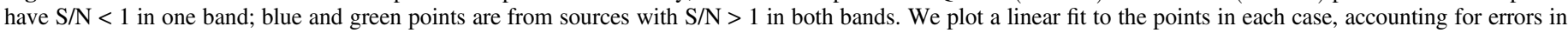

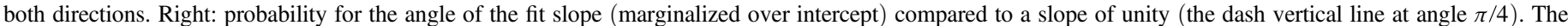
probability of the difference between the angles is also shown with a dotted line.

\subsubsection{QUIET Versus $20 \mathrm{GHz}$ Polarization}

Of the 476 AT20G sources included in our Q-band analysis, 67 have a $20 \mathrm{GHz}$ polarization listed in the catalog. For Wband there is a largely overlapping group of 69 sources. We separate these sources into four groups according to the following criteria: (1) sources for which both AT20G and QUIET set an upper limit on polarization, (2) sources for which AT20G sets an upper limit and QUIET detects, (3) sources AT20G detects and for which QUIET sets an upper limit, and (4) sources which both AT20G and QUIET detect. The last three groups-which include at least one detectionare plotted in Figure 9. As in Figure 6 (which compared to $20 \mathrm{GHz}$ intensity), we see no clear correlation between $20 \mathrm{GHz}$ polarization and our polarization measurements.

\subsubsection{SED for Bright Sources}

For the ten sources with $\mathrm{S} / \mathrm{N} \geqslant 3$ in either of the QUIET bands, we plot in Figure 10 the SEDs combining our measurements and those from the literature. All sources have $20 \mathrm{GHz}$ intensity from the AT20G survey, and many have 5 and $8 \mathrm{GHz}$ total intensity. Many of these bright sources also have some polarization information from AT20G. We plot WMAP and Planck total intensity measurements if a matching catalog source can be found within $4^{\prime}$, a generous approximation of the positional uncertainty.

Variability can be significant for these sources, and complicates the interpretation of the SED. Flux variations are often fairly uniform across millimeter bands (Bolton et al. 2006; Franzen et al. 2009; Chen et al. 2013). The change at higher frequency almost always precedes the change at lower frequency and the polarization fraction could be driven higher or lower as a source flares, due to the physical mechanism of the flux variation. In resolved radio sources, these changes are almost always associated with a new component propagating through the core or along a jet (e.g., Blandford \& Königl 1979; Bridle \& Perley 1984; Middelberg \& Bach 2008), changing the balance of emitted flux.

Because QUIET makes deep polarization maps but not deep temperature maps (Section 2.1), we must rely on WMAP and Planck data for intensity measurements of the sources. That these are not contemporaneous complicates our interpretation. To show variability, in Figure 11 we depict measurement of intensity and polarization for AT20GJ042840-375619 (PKS 0426-380; WMAP J0428-3757; also called PLANCK044 G240.73-43.59 and PMN J0428-3756), highlighting the change in the intensity in WMAP measurements over the 9 years of the mission, beginning in the second half of 2001. QUIET took data in 2008-2010, with the Q-band first and the $\mathrm{W}$-band second. The AT20G catalog data were taken from 2004 to 2008. Planck data from the 2013 release covers two sky surveys from 2009 to 2010 . However, unlike WMAP and QUIET, which make long time averages, Planck's scan strategy yields two snapshots of the sources, taken $\sim 6$ months apart, which are then averaged in the catalog.

Ten of our highest $\mathrm{S} / \mathrm{N}$ sources have their SEDs plotted in Figure 10, each with $\mathrm{S} / \mathrm{N} \geqslant 3$ in at least one QUIET band. We expect about 1.3 spurious detections at $\mathrm{S} / \mathrm{N}>3$ for each band, so $2.6 \pm 1.6$ of these SEDs may be selected due to a spurious fluctuation. We plot two combinations of WMAP data, namely values from the nine-year catalog and an average of years 2008-2010, which are contemporaneous to the QUIET observations.

The SEDs are diverse and interesting. Of these bright sources, only Pict A (AT20GJ051926-454554) has a polarization SED that is unambiguously falling. However, we want to be cautious, since W-band's larger errors, in combination with our S/N cut, may cause spurious detections to favor rising SEDs. All four sources that have a total intensity amplitudes above $1 \mathrm{Jy}$ as observed by WMAP and Planck also have a polarization fraction above $1 \%$, and a few have very large polarization fractions. For instance, AT20GJ042840-375619 in W-band has a polarization of $12.1_{-2.2}^{+2.1} \%$ compared to the Planck total intensity catalog value at $100 \mathrm{GHz}$. The polarization fraction compared to $W M A P$ is higher, $19.3_{-4.6}^{+5.7} \%$, but WMAP averages the total intensity over a longer duration. The source AT20GJ010645-403419 has a polarization fraction at Q-band of $11.1_{-3.3}^{+4.1} \%$ compared to Planck, but we do not detect it in W-band. The total intensity measurement in WMAP Q-band is higher, so the comparative polarization fraction is lower, $6.3_{-1.7}^{+1.9} \%$. For Pict A's western lobe (AT20GJ051926-454554), the WMAP and Planck intensity data pick up contributions from the nucleus and eastern lobe, which reduces the effective polarization fraction in $>10^{\prime}$ resolution imaging to $4.1 \pm 0.6 \%$ at Q-band.

Several of the SEDs appear peculiar. For instance, the QUIET polarized flux density is sometimes equal to or greater 

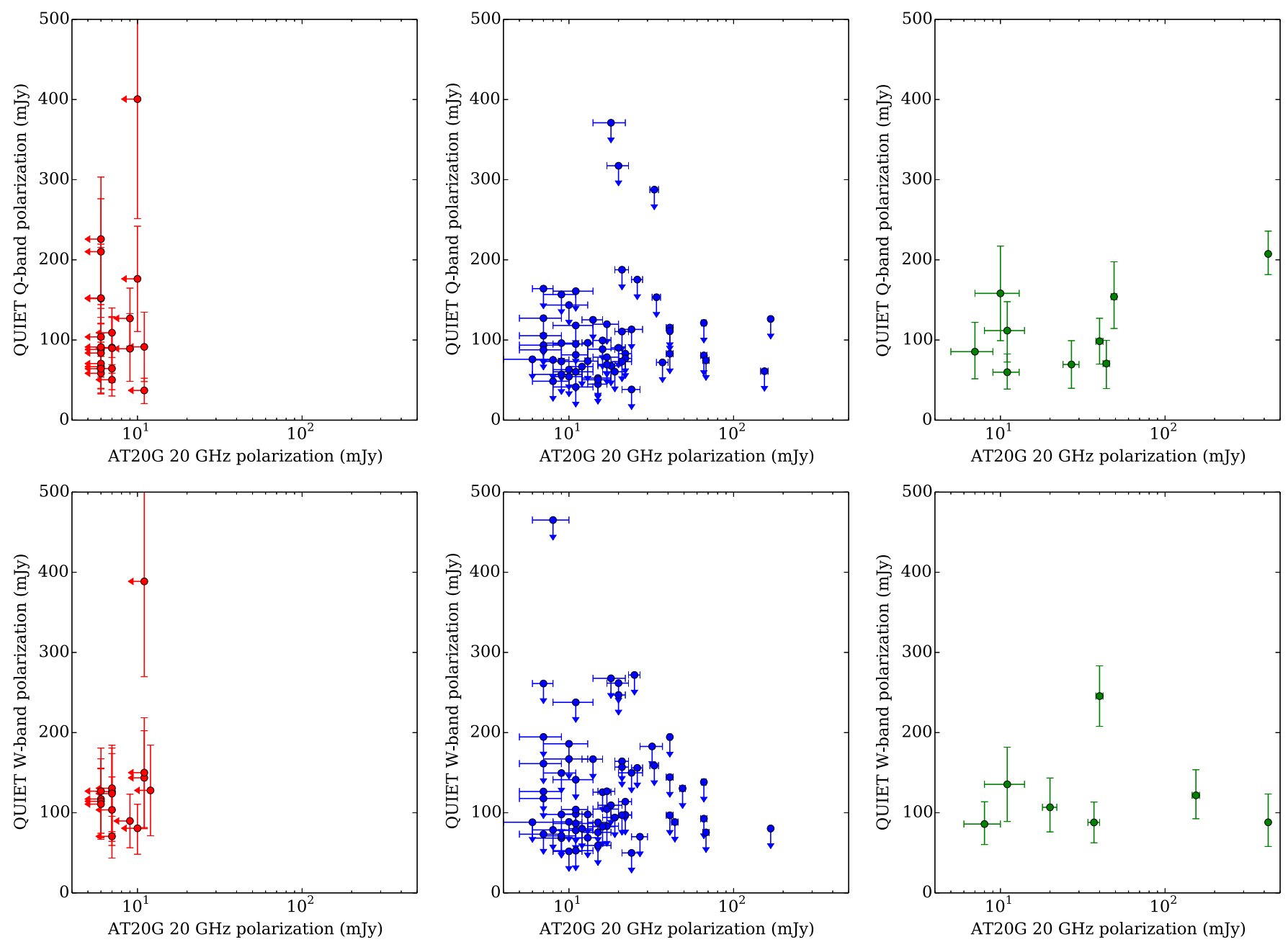

Figure 9. QUIET data vs. $20 \mathrm{GHz}$ polarization. Top row: QUIET Q-band $(43 \mathrm{GHz})$ measurements. Left, limits for AT20G and errors for QUIET. Center, errors for AT20G and limits for QUIET. Right, errors for both AT20G and QUIET. Bottom row: the same but for QUIET W-band $(95 \mathrm{GHz})$.

than the total intensity measurement at 5-20 GHz. Variability could explain this, but the relevant sources are for the most part not listed in the WMAP or Planck catalogs, which implies that the total intensity flux density must be below $\sim 1$ Jy. This in turn would mean that the polarization fractions are very large. Some of these fainter sources may be spurious (such as AT20GJ005645-445102, AT20GJ050838-330853, and AT20GJ113855-465342), because they are significant in only one band. However, AT20GJ123045-312123 is detected with significances of $\mathrm{S} / \mathrm{N}=3.29$ and $\mathrm{S} / \mathrm{N}=2.28$ in the Q- and $\mathrm{W}$ bands, and the probability is $1.1 \%$ that we should encounter in both bands simultaneously a spurious fluctuation this large or larger.

\section{CONCLUSIONS}

We have measured polarization at 43 and $95 \mathrm{GHz}$ at the locations of $\sim 48020 \mathrm{GHz}$-selected radio sources. We have several $\mathrm{S} / \mathrm{N}>3$ detections of polarized emission. Since we know from the $20 \mathrm{GHz}$ data that sources are present, our many upper limits also provide useful constraints for the 43 and $95 \mathrm{GHz}$ polarized source populations. We find no immediately obvious trend between the $20 \mathrm{GHz}$ intensity or polarization and the higher frequency polarization. Our results do not support simple models that assume source populations with uniform SEDs and polarization fractions.

We find similar numbers of high-significance polarization detections at 43 and $95 \mathrm{GHz}$, despite a lower sensitivity in the higher frequency band. This may suggest a flat spectrum in polarization. Several of the bright sources show the same trend. However the $\mathrm{S} / \mathrm{N}$ is too low for most individual sources to allow us to draw firm conclusions from direct band-to-band comparisons of flux densities. The SEDs of bright sources are diverse and interesting, and may in some cases require significant variability to make sense.

The lack of correlation between the Stokes parameters in the bands of our experiment is an interesting result, and we may speculate about the physical explanation. For RM of $10^{2}-10^{4} \mathrm{rad} \mathrm{m}^{-2}$ (e.g., Zavala \& Taylor 2004), the expected Faraday rotation between 43 and $95 \mathrm{GHz}$ is $\sim 0^{\circ} .2-20^{\circ}$, not enough to completely decorrelate the Stokes parameters. Thus the reason may lie in the complications of AGN-powered radio emission, with contributions from the core and the jets as they propagate out into the intergalactic medium and form lobes. Separate episodes of black hole activity can produce distinct energy distributions in the out-going electrons. Turbulent jet interactions with the ambient material can kink the magnetic field lines and alter polarization direction in the synchrotron emission, which dominates at these frequencies. In flat-spectrum radio 

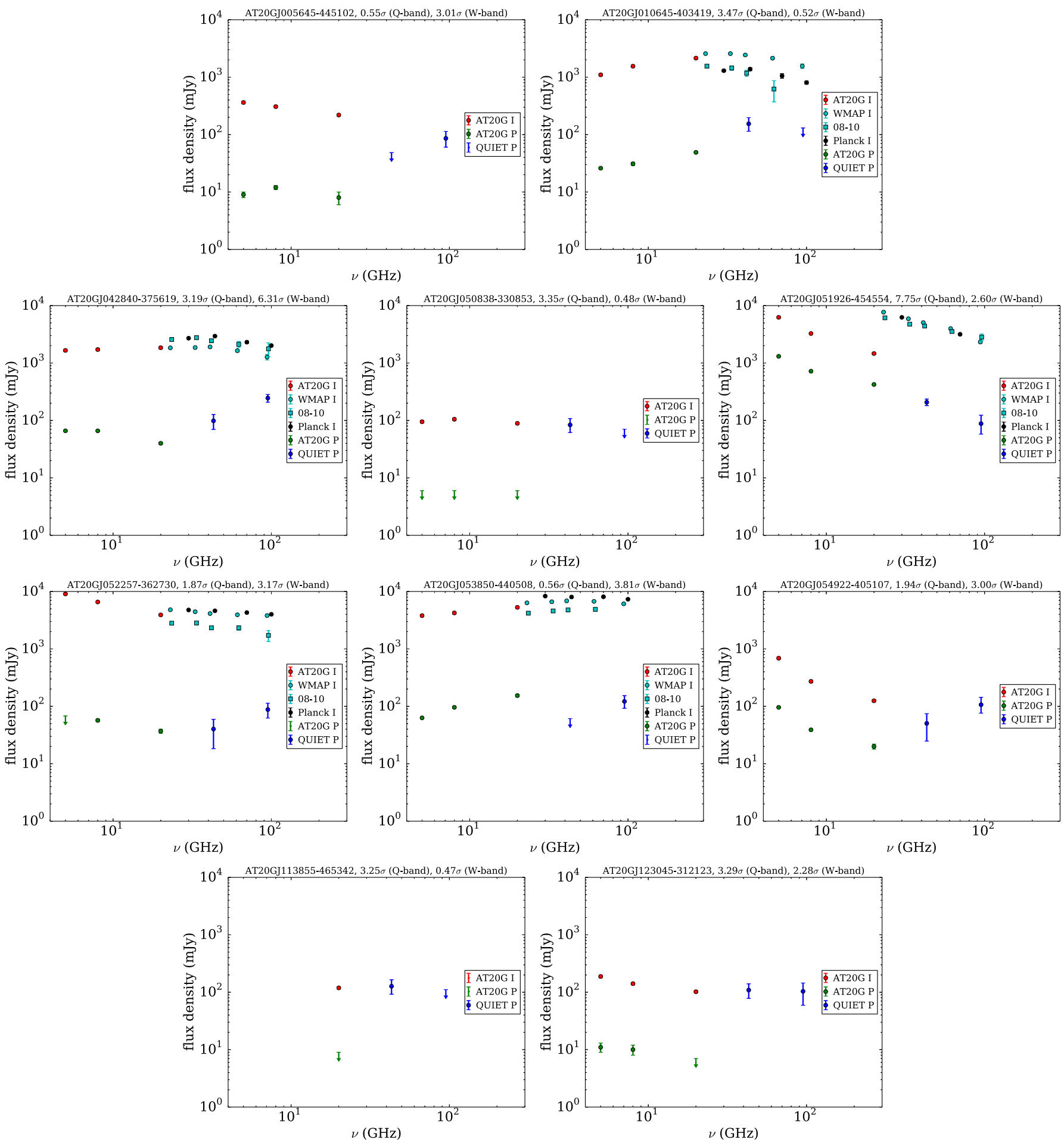

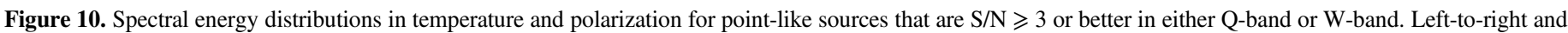

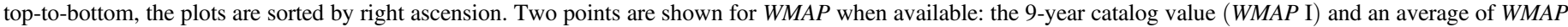
individual year catalogs (2008-2010) overlapping in time with QUIET observations.

quasars and blazars, the emission comes from optically thick portions of the jet close to the core (e.g., de Zotti et al. 2005; Tucci et al. 2011). In many of these sources, QUIET's frequency bands lie near the low-frequency cut off in the SEDs due to selfabsorption (Potter \& Cotter 2013). Thus opacity may be determining the physical region important for emission: higher frequencies probe deeper into the jet material. For steep spectrum sources, on the other hand, the emission comes from the radio lobes, which are optically thin, and therefore differences in the locations where emission is strongest may be due to spatial differences in the populations of energetic electrons. In Cen A, for example, we clearly see that the polarized emission in the lobes comes from different physical locations at different frequencies, with different polarization directions. 


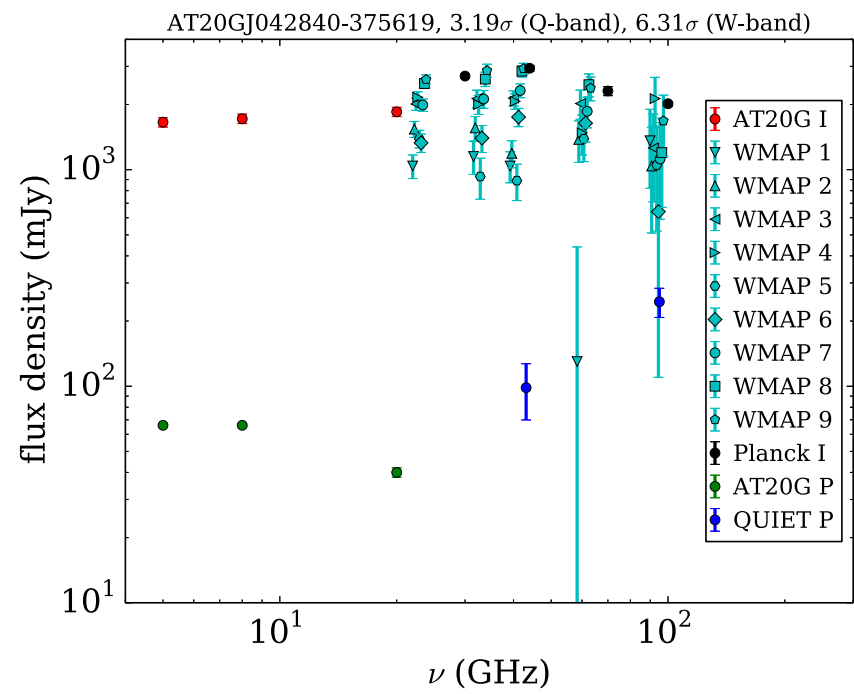

Figure 11. Intensity and polarization measurements for AT20GJ042840375619. WMAP measurements are catalog values for total intensity, by year of WMAP data.

With these observations, we have in hand a probability distribution for the polarization for each source. That allows us to set constraints on properties of the whole population. In the future we will test models of polarized source counts, and to assess the implied impact on $\mathrm{CMB}$ polarization.

Future CMB surveys (e.g., AdvACTPol, ${ }^{30}$ Simons Array, ${ }^{31}$ SPT3G ${ }^{32}$ COrE,$+{ }^{33}$ LiteBIRD $^{34}{ }^{\text {PIXIE }}{ }^{35}$ ) will make even more sensitive polarization measurements over large areas of the sky. The methods presented here for detection and analysis of extragalactic, polarized sources can be readily applied to those data sets, revealing more about the properties of AGN emission.

Bruce Winstein, who led the QUIET project, died in 2011, soon after observations concluded. The project's success owes a great debt to his intellectual and scientific leadership.

Support for the QUIET instrument and operation was provided through the NSF cooperative agreement AST0506648. Support was also provided by NSF awards PHY0855887, PHY-0355328, AST-0448909, AST-1010016, and PHY-0551142; KAKENHI 20244041, 20740158, and 21111002; PRODEX C90284; a KIPAC Enterprise grant; and by the Strategic Alliance for the Implementation of New Technologies (SAINT). This research used resources of the National Energy Research Scientific Computing Center (NERSC), which is supported by the Office of Science of the U.S. Department of Energy under Contract No. DE-AC0205CH11231.

Some work was performed on the Joint Fermilab-KICP Supercomputing Cluster, supported by grants from Fermilab, the Kavli Institute for Cosmological Physics, and the University of Chicago. Some work was performed on the Abel Cluster, owned and maintained by the University of Oslo

\footnotetext{
${ }^{30} \mathrm{http} / / / \mathrm{www} \cdot$ princeton.edu/act/

31 http://cosmology.ucsd.edu/simonsarray.html

32 http://pole.uchicago.edu/

$33 \mathrm{http}: / / \mathrm{www} . c 0 r e-m i s s i o n . o r g$

34 http://litebird.jp/eng/

35 Kogut et al. (2011).
}

and NOTUR (the Norwegian High Performance Computing Consortium), and on the Central Computing System, owned and operated by the Computing Research Center at KEK. Portions of this work were performed at the Jet Propulsion Laboratory (JPL) and California Institute of Technology, operating under a contract with the National Aeronautics and Space Administration. The Q-band modules were developed using funding from the JPL R\&TD program. We acknowledge the Northrop Grumman Corporation for collaboration in the development and fabrication of HEMT-based cryogenic temperature-compatible MMICs.

C.D. acknowledges an STFC Advanced Fellowship, an EU Marie-Curie IRG grant under the FP7 and an ERC Starting Grant (No. 307209). H. K. E. acknowledges an ERC Starting Grant under FP7. A. D. M. acknowledges a Sloan foundation fellowship. J. Z. gratefully acknowledges a South Africa National Research Foundation Square Kilometre Array Research Fellowship.

PWV measurements were provided by the Atacama Pathfinder Experiment (APEX). We thank CONICYT for granting permission to operate within the Chajnantor Scientific Preserve in Chile, and ALMA for providing site infrastructure support. Field operations were based at the Don Esteban facility run by Astro-Norte. We are particularly indebted to the engineers and technician who maintained and operated the telescope: J. Cortés, C. Jara, F. Muñoz, and C. Verdugo.

In addition, we would like to acknowledge the following people for their assistance in the instrument design, construction, commissioning, operation, and in data analysis: A. G. Aitken, C. Baines, P. Bannister, H. Barker, M. R. Becker, A. Blein, A. Brizius, L. Bronfman, R. Bustos, A. Campbell, A. Chandra, S. M. Cho, S. Church, J. Cooperrider, M. Crofts, E. Curry, M. Daly, F. Dejongh, J. Didier, G. Dooley, R. Dumoulin, H. Eide, W. Grainger, J. Goh, P. Hamlington, T. Higuchi, S. Hillbrand, B. Hooberman, K. D. Huff, K. Ishidoshiro, N. Jarosik, M. E. Jones, P. Kangaslahti, D. J. Kapner, E. Komatsu, J. Kristiansen, D. Kubik, R. Lai, C. R. Lawrence, D. Leibovitch, K. Lepo, S. Li, M. Limon, M. Malin, M. McCulloch, J. J. McMahon, O. Montes, D. Moore, M. Nagai, H. Nguyen, G. Nixon, I. O’Dwyer, G. Orellana, S. Osborne, S. Padin, T. J. Pearson, F. Pedreros, A. Perko, L. Piccirillo, J. L. Richards, A. Robinson, D. Samtleben, J. Sanders, D. Sanford, Y. Savon, M. Seiffert, M. Shepherd, K. Smith M. Soria, A. Sugarbaker, D. Sutton, K. Vanderlinde, M. Vidal, L. Volkova, R. Williamson, S. Xenos, O. Zapata, and M. Zaskowski.

Some of the results in this paper have been derived using the HEALPix package (Górski et al. 2005).

\section{REFERENCES}

Agudo, I., Thum, C., Gómez, J. L., \& Wiesemeyer, H. 2014, A\&A, 566, A59 Agudo, I., Thum, C., Wiesemeyer, H., \& Krichbaum, T. P. 2010, ApJS, 189, 1 Agudo, I., Thum, C., Wiesemeyer, H., et al. 2012, A\&A, 541, A111 Antonucci, R. 1993, ARA\&A, 31, 473

Battye, R. A., Browne, I. W. A., Peel, M. W., Jackson, N. J., \& Dickinson, C. 2011, MNRAS, 413, 132

Becker, R. H., White, R. L., \& Helfand, D. J. 1995, ApJ, 450, 559 Begelman, M. C., Blandford, R. D., \& Rees, M. J. 1984, RvMP, 56, 255 Bennett, C. L., Larson, D., Weiland, J. L., et al. 2013, ApJS, 208, 20 BICEP Collaboration et al. PhRvL, 112, 241101

BICEP 2 Collaboration 2014, PhRvL, 112, 21101

Blandford, R. D., \& Königl, A. 1979, ApJ, 232, 34

Bolton, R. C., Chandler, C. J., Cotter, G., et al. 2006, MNRAS, 370, 1556 Bridle, A. H., \& Perley, R. A. 1984, ARA\&A, 22, 319 
Chen, X., Rachen, J. P., López-Caniego, M., et al. 2013, A\&A, 553, A107 Condon, J. J., Cotton, W. D., Greisen, E. W., et al. 1998, AJ, 115, 1693 Curto, A., Tucci, M., González-Nuevo, J., et al. 2013, MNRAS, 432, 728 de Zotti, G., Gruppioni, C., Ciliegi, P., Burigana, C., \& Danese, L. 1999, NewA, 4, 481

de Zotti, G., Ricci, R., Mesa, D., et al. 2005, A\&A, 431, 893

Franzen, T. M. O., Davies, M. L., Davies, R. D., et al. 2009, MNRAS, 400, 995

Górski, K. M., Hivon, E., Banday, A. J., et al. 2005, ApJ, 622, 759

Haslam, C. G. T., Salter, C. J., Stoffel, H., \& Wilson, W. E. 1982, A\&AS, 47, 1

Hirata, C. M., \& Seljak, U. 2003, PhRvD, 68, 083002

Holler, C. M., et al. 2011, arXiv:1111.2702

Jackson, N., Browne, I. W. A., Battye, R. A., Gabuzda, D., \& Taylor, A. C. 2010, MNRAS, 401, 1388

Junkes, N., Haynes, R. F., Harnett, J. I., \& Jauncey, D. L. 1993, A\&A, 269, 29

Kamionkowski, M., Kosowsky, A., \& Stebbins, A. 1997, PhRvD, 55, 7368

King, O. G., Jones, M. E., Blackhurst, E. J., et al. 2014, MNRAS, 438, 2426

Kogut, A., Fixsen, D. J., Chuss, D. T., et al. 2011, JCAP, 7, 25

Larson, D., Dunkley, J., Hinshaw, G., et al. 2011, ApJS, 192, 16

López-Caniego, M., Massardi, M., González-Nuevo, J., et al. 2009, ApJ, 705,868

Massardi, M., Bonaldi, A., Bonavera, L., et al. 2011a, MNRAS, 415, 1597

Massardi, M., Burke-Spolaor, S. G., Murphy, T., et al. 2013, MNRAS, 436, 2915

Massardi, M., Ekers, R. D., Murphy, T., et al. 2011b, MNRAS, 412, 318

Massardi, M., Ekers, R. D., Murphy, T., et al. 2008, MNRAS, 384, 775

Mesa, D., Baccigalupi, C., de Zotti, G., et al. 2002, A\&A, 396, 463

Middelberg, E., \& Bach, U. 2008, RPPh, 71, 066901

Monsalve, R. A. 2010, Proc. SPIE, 7741, 77412M

Montier, L., Plaszczynski, S., Levrier, F., et al. 2015a, A\&A, 574, A135

Montier, L., Plaszczynski, S., Levrier, F., et al. 2015b, A\&A, 574, A136

Murphy, E. J., \& Baum, S. 2014, A Preliminary Summary of The VLA Sky Survey, Tech. Rep. (National Radio Astronomy Observatory, VLASS Survey Science Group) http://science.nrao.edu/science/surveys/vlass/ TheVLASKYSurveyDefinitionPrime.pdf

Murphy, T., Sadler, E. M, Ekers, R. D., et al. 2010, MNRAS, 402, 2403

Okamoto, T., \& Hu, W. 2003, PhRvD, 67, 083002

Page, L., Hinshaw, G., Komatsu, E., et al. 2007, ApJS, 170, 335

Perley, R. A., Roser, H.-J., \& Meisenheimer, K. 1997, A\&A, 328, 12
Planck Collaboration et al. A\&A, 571, A28

Planck Collaboration Int. XXX, et al. 2014, arXiv:1409.5738

Plaszczynski, S., Montier, L., Levrier, F., \& Tristram, M. 2014, MNRAS, 439, 4048

Potter, W. J., \& Cotter, G. 2012, MNRAS, 423, 756

Potter, W. J., \& Cotter, G. 2013, MNRAS, 436, 304

Press, W. H., Teukolsky, S. A., Vetterling, W. T., \& Flannery, B. P. 1992, Numerical Recipes in C: The Art of Scientific Computing (Cambridge: Cambridge Univ. Press)

QUIET Collaboration, Araujo, D., Bischoff, C., et al. 2012, ApJ, 760, 145

QUIET Collaboration, Bischoff, C., Brizius, A., et al. 2011, ApJ, 741, 111

QUIET Collaboration, Bischoff, C., Brizius, A., et al. 2013, ApJ, 768, 9

Quinn, J. L. 2012, A\&A, 538, A65

Readhead, A. C. S., Cohen, M. H., Pearson, T. J., \& Wilkinson, P. N. 1978, Natur, 276, 768

Rubiño-Martín, J. A., Rebolo, R., Aguiar, M., et al. 2012, Proc. SPIE, 8444, $84442 \mathrm{Y}$

Schultz, K. W., \& Huffenberger, K. M. 2012, MNRAS, 424, 3028

Simmons, J. F. L., \& Stewart, B. G. 1985, A\&A, 142, 100

Skrutskie, M. F., Cutri, R. M., Stiening, R., et al. 2006, AJ, 131, 1163

Smith, K. M., Hanson, D., LoVerde, M., Hirata, C. M., \& Zahn, O. 2012, JCAP, 6, 14

Stil, J. M., Keller, B. W., George, S. J., \& Taylor, A. R. 2014, ApJ, 787, 99

The COrE Collaboratione, et al. 2011, arXiv:1102.2181

Tucci, M., Martínez-González, E., Toffolatti, L., González-Nuevo, J., \& de Zotti, G. 2004, MNRAS, 349, 1267

Tucci, M., Martínez-González, E., Vielva, P., \& Delabrouille, J. 2005, MNRAS, 360, 935

Tucci, M., \& Toffolatti, L. 2012, AdAst, 2012, 52

Tucci, M., Toffolatti, L., de Zotti, G., \& Martínez-González, E. 2011, A\&A, 533, A57

Urry, C. M., \& Padovani, P. 1995, PASP, 107, 803

Vaillancourt, J. E. 2006, PASP, 118, 1340

Vidal, M., Leahy, J. P., \& Dickinson, C. 2014, arXiv:1410.4436

Wright, E. L., Chen, X., Odegard, N., et al. 2009, ApJS, 180, 283

Zaldarriaga, M., \& Seljak, U. 1997, PhRvD, 55, 1830

Zavala, R. T., \& Taylor, G. B. 2002, ApJL, 566, L9

Zavala, R. T., \& Taylor, G. B. 2003, ApJ, 589, 126

Zavala, R. T., \& Taylor, G. B. 2004, ApJ, 612, 749 